\title{
Design Tradeoffs and Challenges in Practical Coherent Optical Transceiver Implementations
}

\author{
Damián A. Morero, Mario A. Castrillón, Alejandro Aguirre, Mario R. Hueda, and Oscar E. Agazzi, Life Fellow, IEEE
}

(Tutorial Review)

\begin{abstract}
This tutorial discusses the design and ASIC implementation of coherent optical transceivers. Algorithmic and architectural options and tradeoffs between performance and complexity/power dissipation are presented. Particular emphasis is placed on flexible (or reconfigurable) transceivers because of their importance as building blocks of software-defined optical networks. The paper elaborates on some advanced digital signal processing (DSP) techniques such as iterative decoding, which are likely to be applied in future coherent transceivers based on higher order modulations. Complexity and performance of critical DSP blocks such as the forward error correction decoder and the frequencydomain bulk chromatic dispersion equalizer are analyzed in detail. Other important ASIC implementation aspects including physical design, signal and power integrity, and design for testability, are also discussed.
\end{abstract}

Index Terms-ASIC, chromatic dispersion equalization, CMOS implementation, DSP, FEC, iterative optical receivers, optical fiber, reconfigurable coherent transceivers, VLSI.

\section{INTRODUCTION}

$\mathbf{T}$ HE combination of digital signal processing (DSP), advanced CMOS VLSI technology, and coherent optical transmission has revolutionized optical communications and it has enabled major increases in speed, capacity, spectral efficiency and flexibility of optical transmission, as well as major cost reductions [1]-[4]. Transmission at 100 Gigabits per second $(\mathrm{Gb} / \mathrm{s})$ with dual polarization (DP) quadrature phase shift keying (QPSK) coherent systems (i.e., symbol rate $\approx 32$ Gigabaud, GBd) has reached maturity and widespread deployment. $200 \mathrm{~Gb} / \mathrm{s}$ DP 16-quadrature amplitude modulation (16-QAM) coherent transceivers are commercially available today. Semiconductor industry is currently engaged in the ASIC develop-

Manuscript received June 15, 2015; revised July 26, 2015; accepted August 13, 2015. Date of publication August 18, 2015; date of current version January 24, 2016.

D. A. Morero is with the ClariPhy Argentina S.A., Bloque Suquía, Complejo Capitalinas, Córdoba X5000SFV, Argentina, and also with the Universidad Nacional de Córdoba - CONICET, Córdoba X5016GCA, Argentina (e-mail: dmorero@efn.uncor.edu).

M. A. Castrillón and M. R. Hueda are with the Universidad Nacional de Córdoba - CONICET, Córdoba X5016GCA, Argentina (e-mail: acastrillon@ efn.uncor.edu; mhueda@com.uncor.edu).

A. Aguirre is with the ClariPhy Argentina S.A., Bloque Suquía, Complejo Capitalinas, Córdoba X5000SFV, Argentina (e-mail: alejandro. aguirre@clariphy.com.ar).

O. E. Agazzi is with the ClariPhy Communications, Inc., Irvine, CA 92618 USA (e-mail: oscar.agazzi@clariphy.com).

Color versions of one or more of the figures in this paper are available online at http://ieeexplore.ieee.org.

Digital Object Identifier 10.1109/JLT.2015.2470114 ment of transceivers for $400 \mathrm{~Gb} / \mathrm{s}$ and soon it will move to $1000 \mathrm{~Gb} / \mathrm{s}(1 \mathrm{~Tb} / \mathrm{s})$. A way to achieve immediately $400 \mathrm{~Gb} / \mathrm{s}$ rate is to transmit on two wavelengths at $200 \mathrm{~Gb} / \mathrm{s}$ each [1]. Key drivers of coherent technology today are: (i) Increases in speed, (ii) Reduction of power dissipation, and (iii) Flexibility in modulation formats, data rates, coding gain (CG), host-side protocols, etc. [5]. The latter functionality is a fundamental aspect of reconfigurable transceivers, which are key components of software defined optical networks (SDON) [6], [7].

This article presents tradeoffs in the design and implementation of coherent optical reconfigurable transceivers. Algorithmic and architectural options and tradeoffs between performance and complexity are presented and candidate DSP algorithms for next generation coherent transceivers are discussed. As an example, we discuss the joint iterative detection and decoding (JIDD) algorithm proposed in [8]. This algorithm has been found to be effective in compensating laser phase noise, laser frequency fluctuations, and fiber nonlinearities [9].

The telecommunications industry is migrating from static networks with little flexibility to SDON [10], [11]. The motivation for this paradigm shift is that large increases in traffic cannot be supported by static increases in network capacity. The usable bandwidth in fibers is limited and increases in spectral efficiency come at the cost of reduced reach. As a result, a homogeneous increase in data rate or spectral efficiency is not economically viable. Furthermore, telecommunications companies face an increasingly heterogeneous and dynamic environment in optical transport networks (OTN). This includes, among other parameters, connection lengths, bandwidth requirements, and connection hold times. Another reason for this heterogeneous and dynamic environment is the migration to flexible grid dense wavelength division multiplexing (DWDM) links [11]. Supporting elastic or SDONs requires transceivers that support adaptation of at least the following parameters and functionality: modulation format, symbol rate, channel spacing, and forward error correction (FEC) overhead and CG [1], [7], [12]. By adapting these and other parameters it is possible to achieve excellent power dissipation/reach/data rate tradeoffs. The same transceiver can operate in metro, long haul, ultra long haul and submarine links with optimal performance and high power efficiency. Towards this end, FEC codes with medium-high net coding gains (NCG) (e.g., $N C G=[9-13] \mathrm{dB}$ ) at a bit error rate (BER) of $10^{-15}$ and variable overhead $(\mathrm{OH})($ e.g., $\mathrm{OH}=[7-60] \%)$ are mandatory (e.g., see [13] and references therein). Furthermore, the demand for higher symbol rates (e.g., 45 or $64 \mathrm{GBd}$ ) on long fiber links 
prompts the implementation of complex and flexible bulk chromatic dispersion (BCD) equalizers capable of compensating a wide range of chromatic dispersion (e.g., $[0-250] \mathrm{ns} / \mathrm{nm}$ ) [4], [14], [15]. Complexity and power consumption of high performance FEC and BCD blocks are major challenges for practical implementations of commercial devices. ${ }^{1}$

In this paper we address practical aspects of the architecture of commercial coherent transceivers for metro, long haul, and submarine fiber optic communications. We focus the analysis on performance-complexity tradeoffs of the most critical DSP blocks: BCD equalizer and FEC decoder. Implementation topics such as layout, energy efficiency, wiring, signal and power integrity issues, testability, etc., are also presented. Since these factors have a significant impact on performance, it is crucial to take them into account during the design and implementation of the transceiver.

The rest of this paper is organized as follows. Section II discusses architectural tradeoffs as well as some promising DSP techniques. Section III analyzes performance-complexity tradeoffs of the most critical DSP blocks in coherent transceivers, while Section IV addresses practical implementation topics. Finally, concluding remarks are given in Section V. To facilitate reading, frequently used abbreviations are listed in Table I.

\section{ARChitectural TRADEOFFS}

Optical network operators want to achieve the best possible tradeoff between spectral efficiency and reach. On short fibers, where the optical signal-to-noise ratio (OSNR) is high, they want to be able to transmit more bits per unit bandwidth by selecting larger constellations such as 16-QAM, 32-QAM or 64-QAM. But on longer fibers where the OSNR is much lower, they want to be able to switch to smaller constellations such as QPSK or even binary phase shift keying (BPSK) to be able to close the link at the expense of a reduced data rate. Other tradeoffs can be achieved by controlling the code rate or overhead and the CG, varying the symbol rate, etc. If the transceiver can support this type of programmability, it is also possible to achieve the best tradeoff between performance and power dissipation. Moreover, the same transceiver can be used in very different applications, ranging from metro to long haul, ultra long haul or submarine, by just reconfiguring it appropriately. This kind of reconfigurable transceivers is a key component of SDON. In the following we analyze some tradeoffs in the design of the most critical blocks of reconfigurable coherent transceivers.

\section{A. Practical Architecture of Reconfigurable Transceivers}

Reconfigurable transceivers support link adaptation, where transmission parameters such as modulation and coding are adjusted to take advantage of prevailing channel conditions. In this way it is possible to achieve excellent power dissipation/reach/data rate tradeoffs. A related concept is that of sliceable transceivers [7], where, for example, a $400 \mathrm{~Gb} / \mathrm{s}$ transceiver can be sliced into three virtual transceivers with rates 200, 100

\footnotetext{
${ }^{1}$ Development of high speed, high resolution analog-to-digital and digital-toanalog converters (i.e., ADC and DAC) is also a difficult task (e.g., see [16]).
}

TABLE I

LIST OF COMMONLY USED ABBREVIATIONS

\begin{tabular}{|c|c|}
\hline ASIC & Application-Specific Integrated Circuit \\
\hline $\mathrm{B} 2 \mathrm{~B}$ & Back to Back \\
\hline $\mathrm{BCD}$ & Bulk Chromatic Dispersion \\
\hline BER & Bit Error Rate \\
\hline BICM & Bit Interleaved Coded Modulation \\
\hline BPS & Blind Phase Search \\
\hline BPSK & Binary Phase Shift Keying \\
\hline $\mathrm{CDE}$ & Chromatic Dispersion Estimation \\
\hline CG & Coding Gain \\
\hline CPR & Carrier Phase Recovery \\
\hline CTS & Clock Tree Synthesis \\
\hline DFT & Design for Testability \\
\hline DP & Dual Polarization \\
\hline DSP & Digital Signal Processing \\
\hline DWDM & Dense Wavelength Division Multiplexing \\
\hline ECPR & Explicit Carrier Phase Recovery \\
\hline FEC & Forward Error Correction \\
\hline FFE & Feedforward Equalizer \\
\hline FFT & Fast Fourier Transform \\
\hline FTN & Faster-Than-Nyquist \\
\hline HD & Hard Decision \\
\hline IFFT & Inverse Fast Fourier Transform \\
\hline JIDD & Joint Iterative Detection and Decoding \\
\hline LC-CM & Low Complexity Coded Modulation \\
\hline LDPC & Low Density Parity Check \\
\hline NCG & Net Coding Gain \\
\hline $\mathrm{OH}$ & Overhead \\
\hline OSNR & Optical Signal-to-Noise Ratio \\
\hline PDN & Power Distribution Network \\
\hline PMD & Polarization Mode Dispersion \\
\hline QAM & Quadrature Amplitude Modulation \\
\hline QPSK & Quadrature Phase Shift Keying \\
\hline RAM & Random Access Memory \\
\hline SD & Soft Decision \\
\hline SDD & Soft Decision Demapper \\
\hline SDON & Software Defined Optical Networks \\
\hline SQRT-RC & Square Root Raised Cosine \\
\hline SNR & Signal-to-Noise Ratio \\
\hline STA & Static Timing Analysis \\
\hline TPC & Turbo Product Codes \\
\hline UQ & Uniform Quantization \\
\hline VCD & Value Change Dump \\
\hline
\end{tabular}

TABLE II

RECONFIGURABLE PARAMETERS

\begin{tabular}{ll}
\hline \hline Parameter & \multicolumn{1}{c}{ Values } \\
\hline Symbol Rate & $10-32 \mathrm{GBd}$ \\
Modulation Schemes (diff/no-diff) & DP-BPSK, DP-QPSK, DP-8-QAM, \\
& DP-16-QAM \\
Line-side SD-FEC (Gain [dB]/Overhead [\%] $)$ & LDPC (11.3/20, 11.2/18, 11.1/16) \\
Line-side HD-FEC (Gain [dB]/Overhead [\%]) & RS $(8 / 6.67)$ \\
Pilot Symbol Overhead & $0-5 \%$ \\
CD Compensation Capability & $2-250 \mathrm{~ns} / \mathrm{nm}$ \\
Mean PMD Compensation Capability & $10-50 \mathrm{ps}$ \\
Polarization State Tracking & $10-100 \mathrm{kHz}$ \\
Host Protocol & $100 \mathrm{G} \mathrm{Ethernet,} \mathrm{OTU3,} \mathrm{OTU4}$ \\
\hline \hline
\end{tabular}

and $100 \mathrm{~Gb} / \mathrm{s}$. Table II shows some examples of programmable parameters in a reconfigurable transceiver.

Fig. 1 describes the egress path (transmit path from the client towards the optical fiber) of a typical reconfigurable transceiver available today. Data is received from the host on up to 20 lanes 


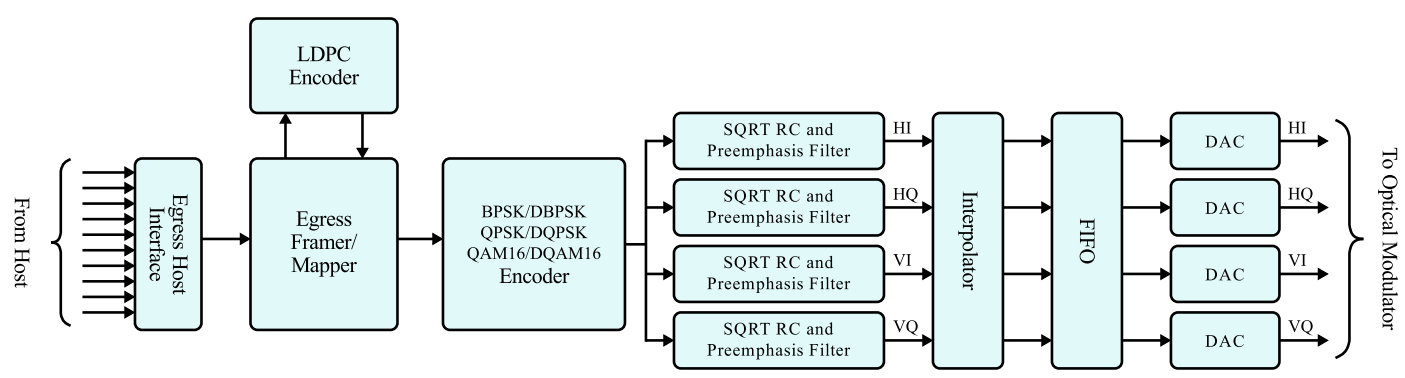

Fig. 1. Block diagram of the egress path.

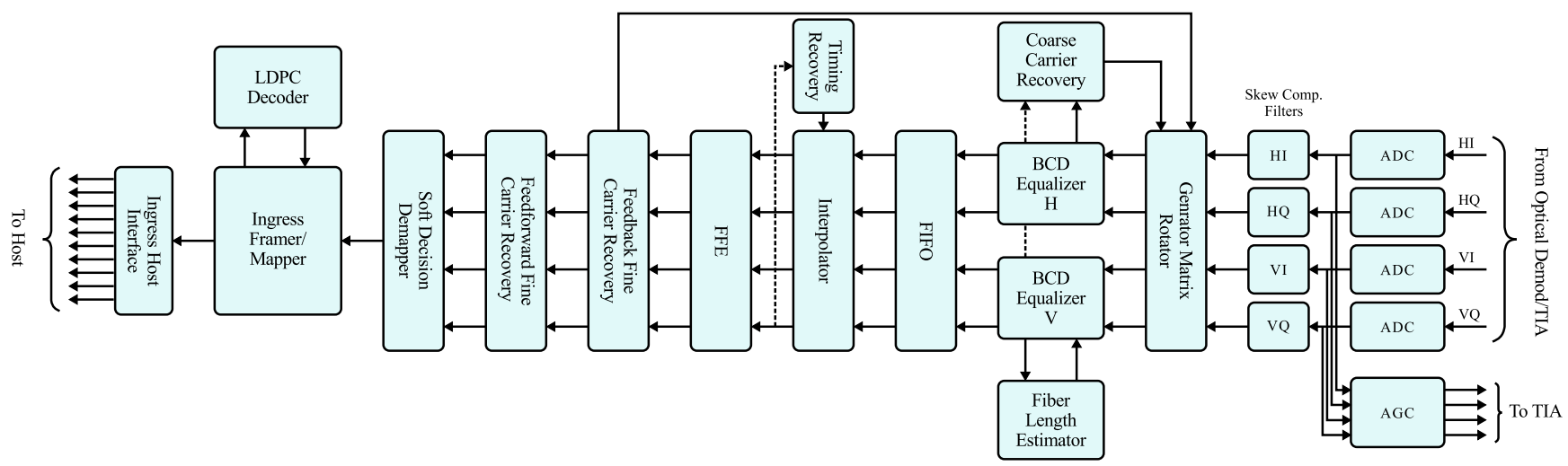

Fig. 2. Block diagram of the ingress path.

at $10 \mathrm{~Gb} / \mathrm{s}$ per lane, and assembled in OTU4 Frames, according to the OTN standards. The data is then modulated in one of various user selectable modulation formats, such as BPSK, QPSK or 16-QAM, either differential or non-differential. Then the symbols are filtered by spectral shaping filters having a square root raised cosine response (SQRT-RC), possibly combined with a pre-emphasis response (necessary to pre-compensate for the attenuation of the electrical circuits connecting the chip with the optical modulator). The sampling rate of these filters is typically twice the symbol rate, but it can be adapted by digital interpolation to a different sampling rate in the DACs, chosen to optimize analog design. The outputs of the transmitter are four signals representing the in-phase and the quadrature components of the horizontal and vertical polarizations. Therefore the optical signal is modulated in both phase and polarization.

Fig. 2 describes the ingress path (receive path from the optical fiber towards the client). The optical signal is converted to electrical and it enters the transceiver as four lanes representing the in-phase and quadrature components of the two polarizations. These are sampled and digitized by four high speed ADCs, sampling typically at a rate of between 55 and $64 \mathrm{GHz}$. After some pre-processing, ${ }^{2}$ the signal is passed to the BCD equalizer, which estimates the chromatic dispersion of the link

\footnotetext{
${ }^{2}$ The preprocessing before $\mathrm{BCD}$ includes: retiming and demultiplexing to reduce the clock rate for parallel processing, compensation of demodulator skews and angular errors, and coarse carrier frequency offset compensation.
}

through the fiber length estimator (FLE) block, and compensates the chromatic dispersion of several thousand kilometers of fiber. SQRT-RC filtering is also implemented in the BCD block. Typical values of the sampling rate in the BCD filter could be in the range of $1.5 / T$ to $1.75 / T$. A FIFO is used to cross from the clock domain of the BCD filter to that of the feedforward equalizer (FFE). The interpolator resamples the signal to $2 / T$ and adjusts the sampling phase under control of the timing recovery (TR) block. TR synchronizes the local clock to the clock of the transmitter at the opposite end of the fiber [17]. It also adjusts the phase to maximize the signal-to-noise ratio (SNR). Then the signal is passed to the FFE, which compensates for polarization mode dispersion (PMD) and other forms of intersymbol interference (ISI), and demultiplexes the polarizations [18]. Then the carrier phase recovery (CPR) block eliminates any residual carrier not eliminated by the local oscillator and compensates phase noise [19], [20], and finally the signal is detected by a soft decision demapper (SDD). The SDD is a generalization of a simple slicer. Instead of hard decisions (HDs), it computes soft decisions that represent the log likelihood ratio of a 0 versus a 1 . This information is used by the soft decision (SD) FEC decoder to achieve higher CG than a traditional hard decision FEC decoder [21]. Finally, the OTU4 frames are identified, the payload is extracted, errors are corrected by the soft decision FEC decoder (e.g., a low density parity check (LDPC) decoder) and the bits are passed to the client through the host interface. 


\section{B. DSP Tradeoffs}

1) Carrier Phase Recovery: CPR is a key function of intradyne coherent optical QPSK/M-QAM receivers [19], [22]. In these devices, CPR algorithms are required to track effects such as phase noise as well as laser frequency fluctuations introduced by mechanical vibrations or other sources, including power supply noise [23]. Phase noise constitutes one of the major factors that limit the performance of coherent transceivers. Phase noise may exist as a result of the nonzero linewidth of the transmit laser or the local oscillator. It is also introduced by self and cross phase modulations (SPM/XPM) in DWDM systems [24]-[27]. Feedforward phase estimation schemes such as the Viterbi Viterbi (VV) or blind phase search (BPS) algorithms have been widely used as a result of their good laser linewidth tolerance and feasibility for parallel implementation [19], [22]. These schemes can be used in combination with feedback CPR techniques in order to improve the tracking of short-term frequency instabilities of the lasers [20]. Alternatively, a JIDD technique with pilot symbols has been found to provide a better performance than traditional CPR solutions in the presence of laser phase noise, fiber nonlinearities, and frequency fluctuations [9]. ${ }^{3}$ However, complexity reduction of the JIDD receiver is still required for it to be applied in commercial devices. In DWDM systems with high XPM-induced phase noise, a pilot $\mathrm{RF}$ tone inserted at the edge of the band in the transmitted signal can be exploited by the receiver to extract a reference for the carrier recovery system [28], [29]. Since the pilot tone is affected by the same phase noise as the signal, the extracted RF tone can be used to compensate the carrier phase noise. A pilot RF tone could also be combined with the JIDD technique to improve the decoding process and avoid the overhead of the pilot symbols typically used in non-differential modulation systems.

2) Chromatic Dispersion Equalization: As we shall show in Section III, reducing the power consumption of the chromatic dispersion equalizer is a challenge in ultra long haul or submarine transceivers required to compensate CD up to 250$300 \mathrm{~ns} / \mathrm{nm}$. Another challenge in all applications of coherent transceivers is achieving high accuracy and fast convergence of the chromatic dispersion estimation (CDE) algorithm [30], [31]. The accuracy of frequency domain CDE techniques based on the timing tone level decreases in the presence of spectral shaping with low roll-off factor. Accuracy and speed of convergence of CDE algorithms based on time domain cost functions depends on the modulation format [30] and may degrade in the presence of high noise and residual channel dispersion (e.g., high-order PMD or controlled ISI in faster-than-Nyquist (FTN) systems [32]). Exhaustive computer simulations are necessary to ensure reliable operation of CDE.

3) Nonlinear Compensation: Nonlinear compensation using digital back propagation has been extensively discussed in the literature [29], [33]-[36]. Some reduced complexity implementations of back propagation have been proposed, however complexity remains very high. A lower complexity technique called perturbation based precompensation has been proposed [37], [38]. This technique is based on computing long sums of

${ }^{3}$ Additional discussion on iterative receivers is provided in Section II-E. cross products of transmitted symbols. The terms are weighted by channel-dependent coefficients. Although the number of terms involved in the summations could be large, complexity is not high because the cross products are essentially logic operations which are efficiently implemented with a few gates. The main challenge with this technique is that the coefficients are not easily determined and there is no known way to make them adaptive.

4) Spectral Shaping: A new standard, called the flexible grid, enables the allocation of fine-grained units of bandwidth to each wavelength. Spectral shaping aims at reducing the bandwidth of the channels with the objective of packing as many channels as possible in a given optical fiber bandwidth, taking advantage of the flexible grid. Conversely, spectral shaping allows maximizing the data rate transmitted on a given bandwidth, such as the typical $50 \mathrm{GHz}$ DWDM channels. As we described previously, spectral shaping is typically implemented using SQRT-RC filters and requires DAC at the TX side. Customers are interested in using roll-off factors as low as possible (e.g., $<5 \%$ ) in order to maximize the spectral efficiency. This creates challenges for several DSP blocks such as TR where a decrease of the roll-off factor reduces the energy of the timing tone used to recover the clock. Recent works have proposed new TR techniques for Nyquist-shaped signals with small roll-off factor [3], [17]. Further work is required to assess their tolerance in the presence of channel impairments such as high-order PMD or residual CD.

\section{FEC Tradeoffs and Trends}

1) Performance: Let $K$ and $N$ be the code dimension and the length of a block FEC code. The code rate is $R=K / N$ while the overhead is given by $\mathrm{OH}=100 \times(N / K-1)[\%]$. $\mathrm{CG}$ is the SNR difference between the uncoded and coded system required to achieve a certain BER (e.g., 10 ${ }^{-15}$ ) over an ideal BPSK modulated additive white Gaussian noise (AWGN) channel. The NCG defined by

$$
\mathrm{NCG}=\mathrm{CG}+10 \cdot \log _{10}(R),
$$

is used to evaluate the performance of a FEC. Note that the NCG takes into account the SNR penalty caused by the bandwidth expansion required to keep the effective information rate of the coded system.

Fig. 3 shows the NCG versus OH map of the state of the art FECs suitable for high speed optical communication [21], [39]-[51]. Since current commercial FECs use moderate OHs ( $\sim 20 \%)$, an important performance increase is expected in the short term by moving from an $\mathrm{OH}$ of $\sim 20 \%$ to values between $25 \%$ and $60 \% .^{4}$ Note that a further increase of the $\mathrm{OH}$ (i.e., $>60 \%$ ) does not provide significant benefits in NCG. Therefore, new elaborate modulation, coding, and DSP solutions will be required in the medium-long term to improve the performance of optical coherent reconfigurable transceivers. Some examples of the most promising techniques are discussed in Section II-E.

\footnotetext{
${ }^{4}$ In applications with limited bandwidth, such as those which have $50 \mathrm{GHz}$ optical filters, the additional overhead may be absorbed by the use of higher order modulations without increasing the signal bandwidth.
} 


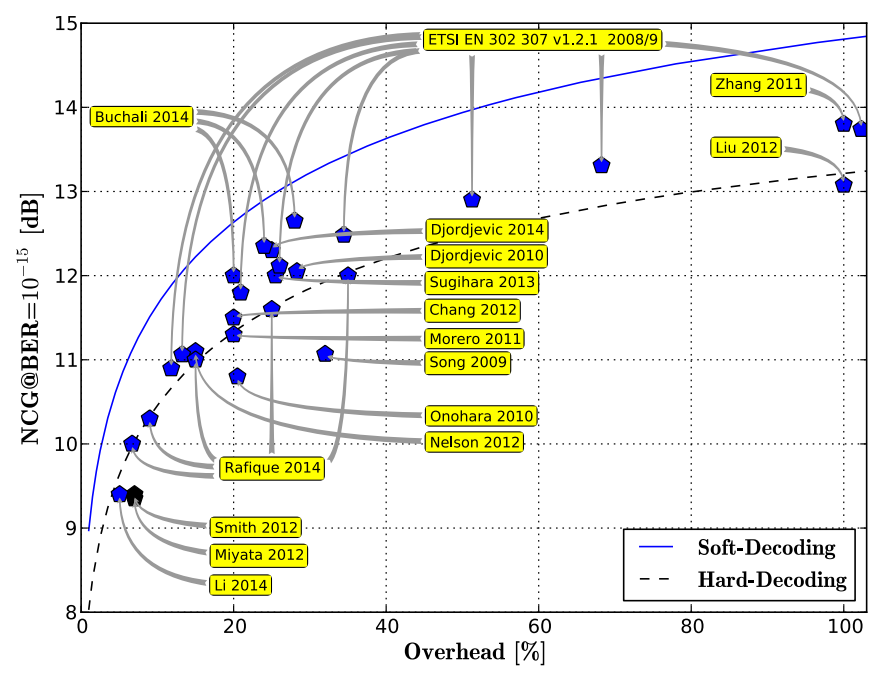

Fig. 3. NCG versus $\mathrm{OH}$ of the state of the art FECs suitable for high speed optical communication [21], [39]-[51].

2) FEC and Pilot Symbols: Constellations with rotational symmetry, such as QPSK or 16-QAM, suffer from certain catastrophic errors called cycle slips, which happen when the carrier phase rotates by a multiple of $90^{\circ}$ [52]. This effect can be mitigated by using differential encoding, but this causes an OSNR penalty in the range of $1 \mathrm{~dB}$. To reduce this penalty, nondifferential modulation can be used in combination with certain known pilot symbols introduced by the transmitter [53]. They allow the receiver to unambiguously determine the carrier phase avoiding or greatly mitigating the effect of cycle slips. Cycle slip correction eliminates most of the penalty of differential modulation. Note that the pilot rate introduces an extra overhead to the coded system. The optimal values of the pilot rate and FEC $\mathrm{OH}$ depend on channel impairments (such as phase noise level) and link configuration (e.g., modulation format). In order to improve spectral efficiency, the $\mathrm{OH}$ of the pilot symbols and FEC code should be among the configurable parameters discussed in Section II.

3) FEC Design and Error Floor: Powerful FEC techniques such as LDPC codes or turbo product codes (TPC) with iterative soft-decision decoding are widely used to provide high NCG at very low BER $\left(10^{-15}\right)$ as required in optical networks. Unfortunately, LDPC and TPC with iterative SD decoding may suffer from error floors at low error rates (e.g., $<10^{-10}$ ) [54]. The error floor problem can be mitigated (or avoided) by a very careful design of both the parity check matrix and the decoder algorithm [42]. Serial code concatenation [55] (SCC) can also be used to combat the error floor problem. Although SCC allows the use of iterative FEC codes optimized for high CG and not for error-floor reduction (e.g., irregular LDPC codes), nonconcatenated FEC schemes may provide a better solution in terms of latency and parameter programmability.

4) Soft and Hard Decoding (SD Versus HD): Soft-decision decoding is able to provide up to $1.5 \mathrm{~dB}$ gain over hard-decision decoding at the expense of a higher implementation complexity. This increase in complexity (and power consumption) of SD decoders cannot be avoided in certain applications (e.g., $\mathrm{OH} \sim 20 \%$ and $\mathrm{NCG} \gtrsim 11.5 \mathrm{~dB}$ ). On the other hand, FEC codes with $\mathrm{OH} \lesssim 15 \%$ and HD decoding are preferred in applications where low power consumption is mandatory. In moderate performance situations (e.g., NCG $\sim[9-11] \mathrm{dB}$ ), a careful comparison between low $\mathrm{OH}-\mathrm{SD}$ and high $\mathrm{OH}-\mathrm{HD}$ is needed to derive a FEC solution that achieves a proper tradeoff between NCG and implementation complexity. The latter topic is discussed in more detail in Section III-D.

5) FEC Latency: FEC latency is of particular importance in applications such as high frequency trading [56] which require very low latency transactions. In these situations the latency introduced by FEC at the transmitter can be avoided by using systematic encoding. However at the receiver the decoder must first collect the whole codeword before correcting the errors. As a consequence, a short codeword length is required to reduce latency. This limits the use of high performance HD-FECs, such as [39], which require large codeword length. Furthermore, in iterative decoders the latency is in general proportional to the number of iterations. Codes with fast convergence are preferable. These codes must be designed to optimize their performance under the constraint of a small number of iterations. The latency caused by iterations can also be reduced by implementing a very fast recursive hardware engine instead of a pipelined chain of concatenated iteration stages. Also note that since short codeword length SD-FECs can provide a performance similar to that of long codeword length HD-FECs, the former becomes an interesting candidate for low latency applications with moderate FEC performance.

\section{Modulation Tradeoffs}

1) Bit Resolution: High order modulation schemes (e.g., 32 or 64 QAM) are required to increase the capacity of limited bandwidth fiber links. This improvement of the fiber capacity is achieved at the expense of an increase of the OSNR. As a result of the implementation penalty caused by several imperfections in practical transceivers, extra OSNR must be added to achieve the expected performance. The limited effective number of bits (ENOB) of ADC/DAC, laser linewidth, and the finite resolution of DSP algorithms are some of the most important limitations in practical VLSI implementation (e.g., see [57] and references therein). In particular, the design of high speed and low power ADCs with moderate resolution (e.g., ENOB 6-7 bit) represents an important challenge for the telecommunication industry. Faster than Nyquist signaling is being considered as an alternative solution to increase the spectral efficiency. This improvement of capacity may be achieved without increasing the modulation order or the sampling rate, thus the requirements for the ADC may be relaxed. Although the complexity of the detection algorithm grows as result of the controlled ISI introduced in FTN, it might be managed by using known high-speed DSP techniques. Therefore, FTN becomes interesting as an alternative to high order modulation based on orthogonal pulses.

2) Bit Mapping: Typically, the constellation points and the bit mapping are designed independently in separate stages. Thus, most of the theory related to the design of constellations focuses on the asymptotic symbol error rate (SER) instead of the BER as a criterion to optimize the design [58]. Once the constellation is designed, bit mapping is achieved by 
using the minimum BER criterion. Better performance can be achieved if the constellation points and the bit mapping are jointly designed [59]. This optimization must be done for each operation point of interest, e.g., at the OSNR threshold of each FEC. Furthermore, the overall performance can be improved if the constellation points, bit mapping, FEC, and interleaving are jointly designed. Towards this end, extrinsic information transfer (EXIT) charts can be used [60]. For coded modulation with a given number of iterations the modulation and the decoder should be jointly designed to maximize the mutual information in the EXIT chart. ${ }^{5}$ On the other hand, for hard-decision FECs (e.g., staircase code [39]), the constellation can be designed by using the distance spectrum [59].

3) Coded Modulation: Coded modulation is the natural evolution of classical constellations to higher dimensions and improved distance properties [61]. It is based on the introduction of interdependencies among sequences of signal points such that not all sequences are possible. As a result, the minimum distance $d_{\text {min }}$ between two possible sequences can be greater than the minimum distance $d_{0}$ in $2 \mathrm{D}$ space. This results in a CG of $10 \log _{10}\left(d_{\min }^{2} / d_{0}^{2}\right)$. In the short term, only low complexity coded-modulation (LC-CM) schemes will be feasible for practical implementation in high-speed optical transceivers. LC-CM combines a simple FEC code with the modulation. To achieve the NCG required in optical networks (e.g., $N C G \gtrsim 11 \mathrm{~dB}$ ), LC-CM must be serially concatenated with a powerful outer code. Tradeoffs must be made between the $\mathrm{OHs}$ of the inner and the outer FEC codes. For instance, LC-CM schemes may be able to provide an extra gain if HD-FECs are used and part of its $\mathrm{OH}$ is shifted to the soft-decision domain of the LC-CM. In the near term LC-CM schemes combined with HD-FEC may be an alternative solution to SD-FEC with moderate gain. When high NCG is required, however, most of the $\mathrm{OH}$ should be in the powerful outer FEC, therefore the improvements achieved by LC-CM are small. Furthermore, in some cases the CG achieved by a LC-CM serially concatenated with a powerful outer code will be lower than the one provided by a traditional bit interleaved coded modulation (BICM) with an SD-FEC and similar $\mathrm{OH}$. This can be observed from Fig. 4 where the BER versus the SNR per bit $\left(E_{b} / N_{0}\right)$ of two LC-CM schemes proposed for high speed optical communication are analyzed: Polarization Switched QPSK (PS-QPSK) [62], [63] and 128 Set Partition QAM (128-SP-QAM) [64]. Comparisons with two BICM systems are also included: $B I C M-1$ and $B I C M-2$. The LDPC code used in BICM-1 is the outer code of the concatenated solution based on LC-CM (i.e., a 20\% LDPC). BICM-2 employs a LDPC code with $\mathrm{OH}$ similar to the one of the serially concatenated scheme (i.e., LC-CM+FEC code used in BICM-1). From Fig. 4 we observe that LC-CM is able to slightly improve the performance respect to BICM-1. However, notice that BICM-2 achieves a better performance than the LC-CM-based solution. Taking into account that the implementation complexities of LC-CM and the high OH-based BICM solutions used in these simulations are similar, we conclude that LC-CM may

\footnotetext{
${ }^{5}$ Although Gray mapping is optimal for non-iterative decoding, it may not be the best solution for iterative decoding of coded modulation schemes.
}

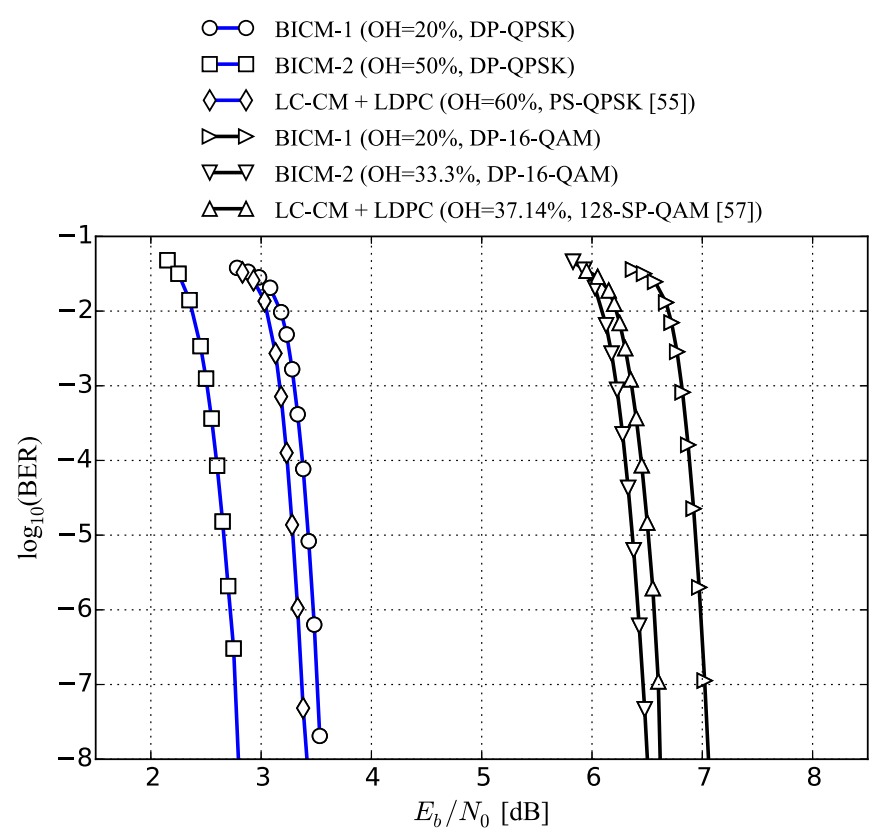

Fig. 4. Performance of BICM schemes versus LC-CM in combination soft decision FECs.

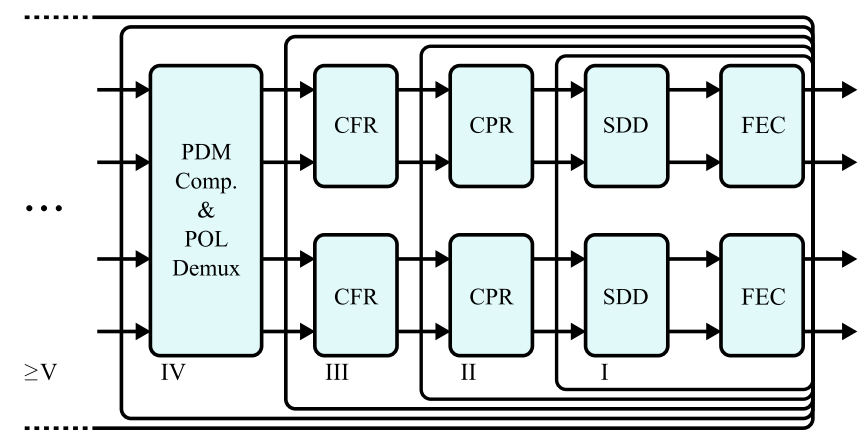

Fig. 5. Iterative receiver architecture: DSP and FEC integration levels.

not be useful in these applications. ${ }^{6}$ In the long term, all the $\mathrm{OH}$ of the FEC should be moved to the inner code in order to maximize the benefits of coded modulation. Towards this end, trellis coded-modulation (TCM) and iterative demodulation and decoding may be adopted. In particular, iterative demodulation and decoding seems to be one of the most promising alternatives in terms of complexity and integration with the other DSP blocks, as we shall discuss in the following section.

\section{E. Advanced DSP Techniques}

1) Iterative Receivers: Performance of coherent receivers can be improved by combining the decoding of the soft decision FEC and demapping, phase and frequency estimation, and feedforward equalization. This can be done by introducing iterations between the FEC decoding and the other blocks as shown in Fig. 5. The first integration step combines modulation and coding (i.e., iterative coded-modulation), as discussed

\footnotetext{
${ }^{6}$ Similar observations have been recently reported in [65] for a 256 points 4D constellation based on the $D_{4}$-lattice $\left(256-D_{4}\right)$ which does not outperform the classical PM-16-QAM when combined with a TPC based FEC.
} 


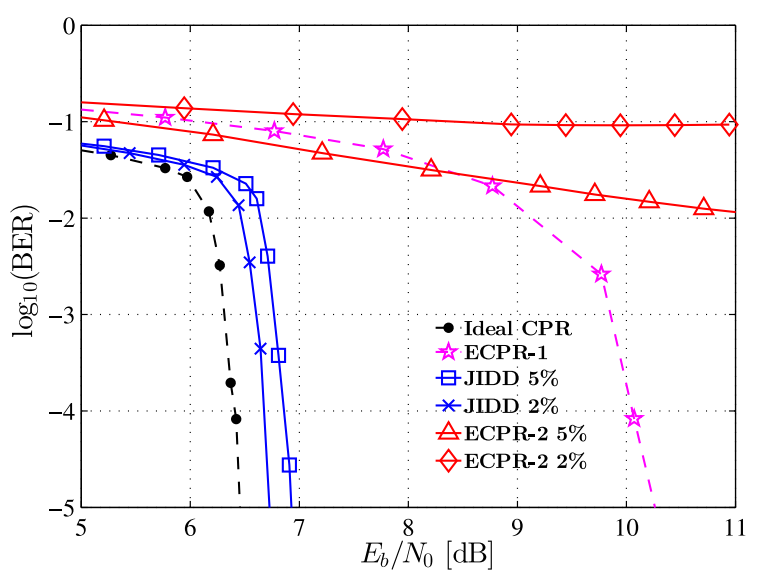

Fig. 6. Post-FEC BER versus $E_{b} / N_{0}$ for DP-16-QAM and $32 \mathrm{GBd}$. Laser linewidth $=500 \mathrm{kHz}$ and frequency fluctuation of $200 \mathrm{MHz}$ at $35 \mathrm{kHz}$ (see [9] for more details).

previously. The second integration step includes the CPR in the iterative algorithm such as the JIDD algorithm proposed in [8]. Fig. 6 shows the performance of JIDD-based optical coherent receivers in the presence of phase noise and laser frequency fluctuations for DP 16-QAM and 32 GBd. The JIDD technique is compared with two state of the art techniques based on an $e x$ plicit CPR (ECPR). The first one denoted ECPR-1 is based on the BPS algorithm combined with differential modulation [19], and the second one denoted ECPR- 2 is based on an interpolation filter aided by pilot symbols followed by a BPS stage [66]. It can be seen that joint iterative decoding and detection greatly outperforms these alternative techniques and continues to work when the others break down. This superiority of the JIDD is still observed even without frequency fluctuations [9].

From the above, it is expected that further integration of other blocks will improve the performance of the transceiver. However, as a result of the prohibitively high implementation complexity of an iterative super-receiver, the integration process is best carried out through local integrations, such as iterative coded-modulation [67], [68], joint state of polarization and carrier phase compensation [69], and stochastic digitalbackpropagation [70].

2) Multiple Carrier Receiver (Superchannels): A superchannel system combines multiple coherent optical carriers in order to create a unified channel of a higher data rate (e.g., see [1] and references therein). In a superchannel system, all optical signals are modulated and multiplexed together at the same site, transmitted and routed together over the optical link, and received at a common site. Superchannels increase the spectral efficiency (i.e., the channel gap is smaller than in DWDM) and the operational scalability. An interesting feature of a superchannel system is the possibility to exploit at the receiver the information of its constituents to implement joint processing [1]. This can be used to simplify some DSP blocks (e.g., carrier frequency recovery) or mitigate certain impairments such as crosstalk between adjacent signals. Taking into account the high interest of the telecommunication industry for sliceable transceivers [7], it is expected that practical joint processing architectures will be required in the medium term.

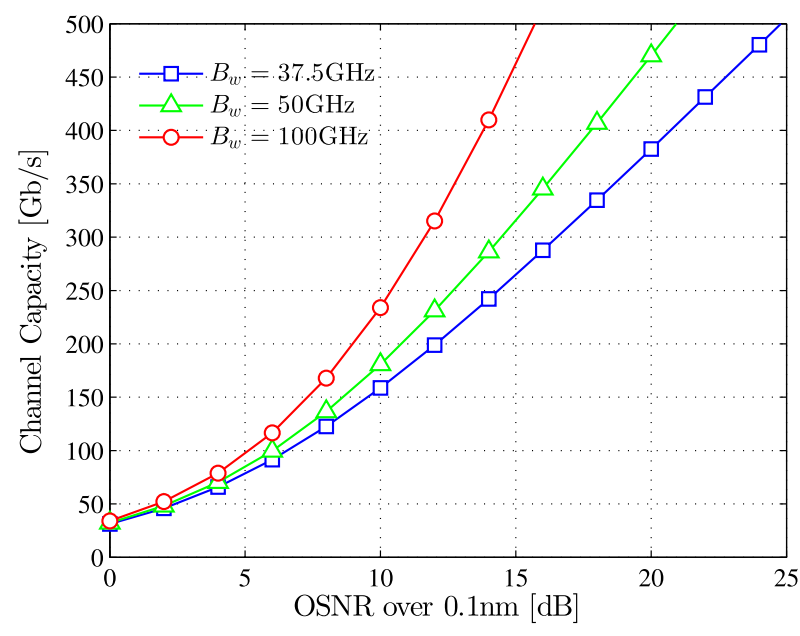

Fig. 7. Channel capacity versus OSNR.

Although superchannels have all the advantages mentioned above, they also require a higher optical component count and increase the total power dissipation, since multiple modulator/demodulator, TIA, and modulator driver sets are required. Other tradeoffs of superchannels are discussed in [71]. One of the solutions to overcome the limitations of superchannels mentioned above is the use of integrated photonics [1]. There is a complementary trend in the industry to move towards higher data rate transmission on a single wavelength [72]. This can be accomplished by increasing spectral efficiency (for example using higher order modulations [73] and/or faster than Nyquist signaling [32],[74]), but this in general comes at the cost of reduced reach. Higher symbol rate transmission with moderate spectral efficiency on a single wavelength is one of the techniques considered for next generation long haul coherent transceivers at data rates of $400 \mathrm{~Gb} / \mathrm{s}$ to $1 \mathrm{~Tb} / \mathrm{s}$ [75]. Of course, higher symbol rate transmission and high spectral efficiency techniques can be used in combination with superchannels to achieve even higher capacity [76].

\section{Performance-Complexity TradeofFs}

\section{A. Fundamental Limits and Implementation Penalty}

It is useful to look at some of the fundamental limits of the performance of the transmission system. In Fig. 7 we plot the channel capacity in $\mathrm{Gb} / \mathrm{s}$ as a function of the OSNR for three channel bandwidths commonly used in optical communications: 100, 50, and $37.5 \mathrm{GHz}$. We assume DP, AWGN, and bandwidth equal to the channel spacing in the DWDM grid. Thus, the classical formula for the channel capacity in Gaussian channels can be expressed in terms of the OSNR over $0.1 \mathrm{~nm}$ as

$$
C=2 B_{w} \log _{2}(1+\underbrace{\operatorname{OSNR} \frac{B_{0}}{B_{w}}}_{\text {SNR }}),
$$

where $B_{w}$ is the channel width and $B_{0}=12.5 \mathrm{GHz}(0.1 \mathrm{~nm})$.

One very important measure of the receiver performance often used by customers to compare different receivers is Back-to- 
TABLE III

EXAMPLES OF B2B OSNR

\begin{tabular}{lccc}
\hline \hline $\begin{array}{l}\text { Data Rate } \\
{[\mathrm{Gb} / \mathrm{s}]}\end{array}$ & $\begin{array}{c}\text { Bandwidth } \\
{[\mathrm{GHz}]}\end{array}$ & $\begin{array}{c}\text { B2B OSNR at } \\
\text { Capacity [dB] }\end{array}$ & $\begin{array}{c}\text { Commercial } \\
\text { Transceivers [dB] }\end{array}$ \\
\hline 100 & 37.5 & 7 & 10.5 \\
200 & 50 & 11 & 17.5 \\
400 & 100 & 13 & N/A \\
\hline \hline
\end{tabular}

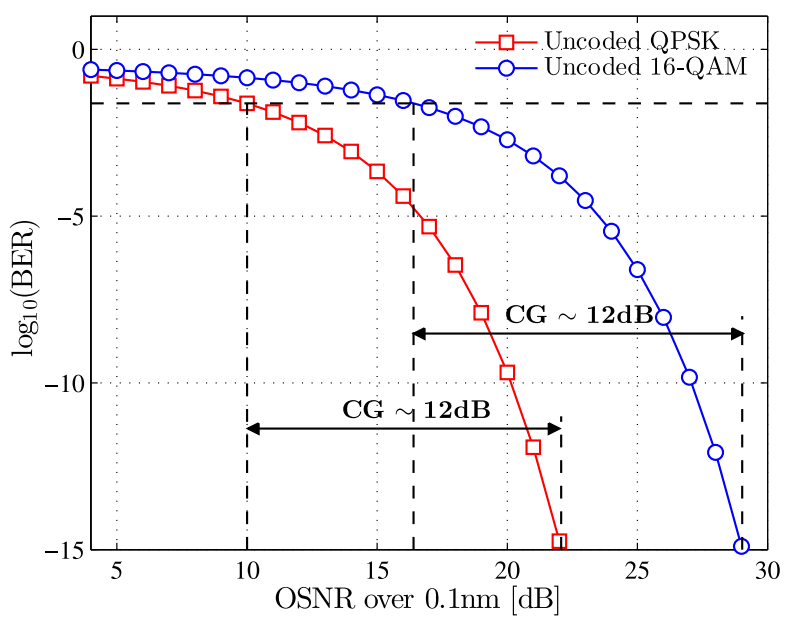

Fig. 8. BER versus OSNR for QPSK and 16-QAM. Symbol rate: $32 \mathrm{GBd}$ and FEC threshold: $2.4 \times 10^{-2}$.

Back (B2B) OSNR. This measure ignores penalties introduced by the channel and it is dominated by the CG of the FEC and (to a lesser extent) by the implementation penalties. It is interesting to find its fundamental limits based on channel capacity. These limits are independent of any particular modulation and coding scheme. They are useful to compare versus specific implementations. Table III compares typical values of OSNR in B2B conditions for state of the art commercial transceivers versus the fundamental limit imposed by the channel capacity given by eq. (2) for various data rates and channel bandwidths.

Unlike in the previous analysis where the ideal B2B OSNR was based on the channel capacity and not on any particular modulation and coding scheme, next we assume specific modulation schemes (QPSK and 16-QAM), and a specific net coding gain $\left(\mathrm{NCG}=11.3 \mathrm{~dB}\right.$ at $\mathrm{BER}=10^{-15}$ and $20 \%$ overhead), and a specific symbol rate ( $32 \mathrm{GBd})$, resulting in a BER threshold for the FEC of $2.4 \times 10^{-2}$ (i.e., $\mathrm{CG}=12 \mathrm{~dB}$ ). From Fig. 8 we verify that the ideal B2B OSNR in these conditions is $10 \mathrm{~dB}$ for QPSK and about $16 \mathrm{~dB}$ for 16-QAM. Comparing the actual B2B OSNR of Table III versus these limits gives an indication of the implementation penalty.

\section{B. Transceiver Complexity}

Table IV shows the relative complexity of blocks in a transceiver that works at $100 \mathrm{~Gb} / \mathrm{s}$ using QPSK modulation, or at $200 \mathrm{~Gb} / \mathrm{s}$ using 16-QAM. The measure of complexity in this table is power dissipation. One interesting conclusion is that 16-QAM doubles the data rate at the expense of only $30 \%$ power increase. Another conclusion is that the blocks that
TABLE IV

RELATIVE COMPLEXITY OF THE TRANSCEIVER BLOCKS

\begin{tabular}{lcc}
\hline \hline Block & 100 Gb/s QPSK & 200 Gb/s 16-QAM \\
\hline FEC Encoder & 0.02 & 0.04 \\
TX DSP & 0.02 & 0.02 \\
BCD Equalizer & 0.21 & 0.21 \\
FFE & 0.14 & 0.14 \\
Carrier Recovery & 0.02 & 0.02 \\
Soft Decision Comp. & 0.03 & 0.03 \\
FEC Decoder & 0.22 & 0.45 \\
Framer/Mapper & 0.03 & 0.06 \\
Miscellaneous & 0.14 & 0.14 \\
AFE & 0.17 & 0.19 \\
Total & 1.00 & 1.30 \\
\hline
\end{tabular}

consume the most power are the BCD equalizer and the LDPC decoder, followed by the FFE and the AFE. Therefore we address performance/complexity tradeoffs for the $\mathrm{BCD}$ and the FEC blocks in some detail in the following sections.

\section{Power Consumption of BCD Equalizers}

Complexity of the BCD equalizer is dominated by the complexity of the fast Fourier transform (FFT) and inverse FFT (IFFT) engines. The most important measure of complexity from a practical standpoint is power dissipation. In practice it is found that power dissipation correlates well with the number of complex multiply-add operations per unit time in the FFT.

$\mathrm{BCD}$ equalizer power estimation is based on scaling a known design which may be in a different technology node and for a different symbol or sampling rate, and it may use a different FFT block size. Scaling is based mainly on the number of complex multiply-add operations per FFT block, and it also accounts for scaling of symbol rate and technology. A good estimate of the $\mathrm{BCD}$ power is given by

$$
P_{\mathrm{BCD}} \approx K_{\mathrm{Tech}} P_{\mathrm{Ref}} f_{B} N_{\mathrm{OPS}},
$$

where $K_{\text {Tech }}$ is the technology scaling factor, $P_{\text {Ref }}$ is a power reference design, $f_{B}$ is the symbol rate; $N_{\mathrm{OPS}}$ is the number of operations per data symbol given by

$$
N_{\mathrm{OPS}}=R \frac{S_{\mathrm{FFT}}+2 N_{\mathrm{FFT}}}{S_{\mathrm{FFT}}-M},
$$

where $R$ is the oversampling factor, $N_{\text {FFT }}$ is the number of operations per FFT block, $S_{\mathrm{FFT}}$ is the FFT block size, and $M$ is the length (in samples) of the CD impulse response. The latter parameter can be roughly approximated by

$$
M=R f_{B} \nu_{m} C_{d}
$$

where $C_{d}$ is the $\mathrm{CD}$ (e.g., $\left.[\mathrm{ns} / \mathrm{nm}]\right)$ and $\nu_{m}$ is the modulated linewidth of the laser given by

$$
\nu_{m}=\frac{(1+\delta) f_{B}}{c} \lambda^{2}
$$

with $\delta, \lambda$, and $c$ being the roll-off factor of the filter, the wavelength (e.g., $1550 \mathrm{~nm}$ ), and the speed of light, respectively.

Equation (4) takes into account the operations required by both FFT and IFFT blocks $\left(2 N_{\mathrm{FFT}}\right)$, as well as the complex 


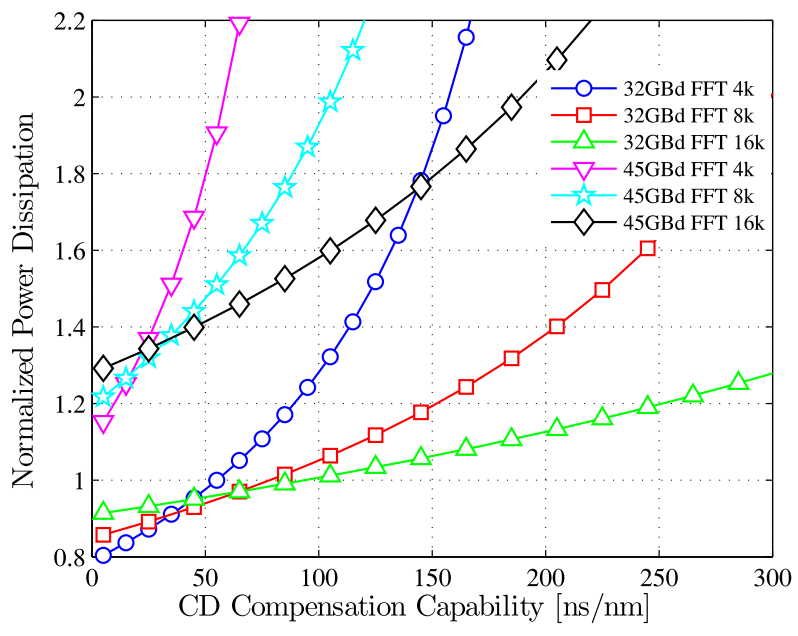

Fig. 9. BCD power versus CD and FFT size. Assumptions: (i) Excess bandwidth $20 \%$, (ii) Oversampling factor 1.6, (iii) Reference design 32GBd symbol rate, $55 \mathrm{~ns} / \mathrm{nm}$ CD compensation capability 4k FFT size, and (iv) Radix 2 FFT.

multiplications used for frequency filtering $\left(S_{\mathrm{FFT}}\right)$. The approximate number of operations per FFT block for radix 2 and radix 4 FFTs are $N_{\mathrm{FFT}}=\frac{S_{\mathrm{FFT}}}{2}\left(\log _{2} S_{\mathrm{FFT}}-1\right)$ and $N_{\mathrm{FFT}}=\frac{3 S_{\mathrm{FFT}}}{4}\left(\log _{4} S_{\mathrm{FFT}}-1\right)$, respectively. There are theoretical bounds for the minimum number of operations in the FFT, such as the Winograd or Heideman bounds [77]. A particular algorithm can be compared to these bounds to evaluate its performance. Many algorithms come close to the bounds, so there is little room for optimization here.

Fig. 9 shows the normalized power dissipation of the $\mathrm{BCD}$ equalizer $\left(\bar{P}_{\mathrm{BCD}}=P_{\mathrm{BCD}} / P_{\mathrm{BCD}, \mathrm{Ref}}\right)$ as a function of $\mathrm{CD}$ compensation capability, FFT block size, and symbol rate. We use $\delta=0.20, R=1.6$, and radix 2 FFT. The reference design $\left(P_{\mathrm{BCD}, \mathrm{Ref}}\right)$ considers a receiver with $32 \mathrm{GBd}$ symbol rate, 55 $\mathrm{ns} / \mathrm{nm}$ CD compensation capability, and 4k FFT block size. The same fabrication technology is assumed for all the cases (e.g., $K_{\text {Tech }}$ is constant). It can be seen that large FFT block sizes are in general more efficient than small blocks, except in the region of very low $\mathrm{CD}$ compensation. For large block size, power dissipation increases modestly as a function of increasing CD. However, small block sizes exhibit a dramatic increase as a result of the loss of efficiency in the computation because of the large size of the overlap block compared to the FFT block. Also, symbol rate has a dramatic effect on the power dissipation.

\section{Complexity of FEC Codes}

Soft decision coding schemes such as LDPC codes or TPCs with large codeword length (e.g., $\gtrsim 10^{4}$ ) are mandatory to achieve near Shannon limit performance. Parallel architectures are required for multigigabit transceivers. ${ }^{7}$ Parallel architectures for LDPC and TPC usually suffer from high layout complexity as a result of the complex interconnection patterns inherent in their iterative decoders. This problem is exacerbated by code rate programmability, required to provide flexibility to SDONs.

\footnotetext{
${ }^{7}$ Serial digital algorithms operating at clock frequencies of hundreds of $\mathrm{GHz}$ are not possible with current CMOS technology (e.g., $16 \mathrm{~nm}$ ).
}

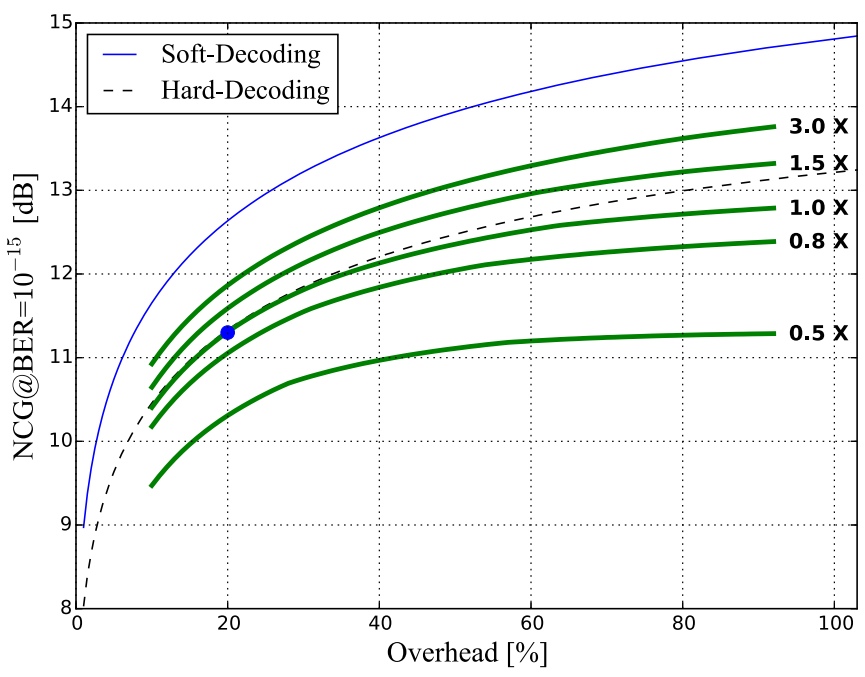

Fig. 10. FEC complexity versus $\mathrm{OH}$ and NCG normalized to the FEC reported in [42], [78], [79] with $N \approx 2 \cdot 10^{4}$ and average parity check matrix column weight 4. Reference (point): $\mathrm{NCG}=11.3 \mathrm{~dB}$ at $\mathrm{BER}=10^{-15}$ with $20 \%$ $\mathrm{OH}$.

To mitigate this problem, a well structured parity check matrix is needed. Although current commercial products show high performance and good flexibility [78], further additional work will be necessary to efficiently implement future generations of FECs with high gains (e.g., NCG $\gtrsim 12 \mathrm{~dB}$ ) and variable code rates. Given the crucial role of FECs with near Shannon limit performance for development of SDON, some practical related aspects of FEC implementation are discussed in the following.

1) Complexity versus FEC Overhead: As observed in Fig. 3, NCGs of current FEC codes closely approach the Shannon capacity. Unfortunately, for a given $\mathrm{OH}$, an important increase in complexity is needed to further increase the NCG by just a fraction of a dB. This can be inferred from Fig. 10, where an estimated normalized complexity of FEC is depicted as a function of the $\mathrm{OH}$ and $\mathrm{NCG}^{8}$. For example, $\mathrm{NCG}$ can be increased from 11.3 to $11.9 \mathrm{~dB}$ with $20 \% \mathrm{OH}$ at the expense of an increase of three times $(\sim 3 \times)$ in complexity. Notice that the same NCG improvement can be achieved by a FEC with $\sim 42 \% \mathrm{OH}$ and lower $(\sim 0.8 \times)$ complexity. Therefore, industry is considering the use of higher OHs as a practical approach to $(i)$ increase the NCG and (ii) reduce the impact on the FEC complexity. It is important to realize that the higher $\mathrm{OH}$ requires an increase of

\footnotetext{
${ }^{8}$ The NCG and the complexity of an LDPC code for fixed codeword length $N$ and average parity check matrix column weight $\bar{w}_{c}$ can be approximated by functions $\Phi(R, i, b)$ and $\mathcal{O}(\nu, R, i, b)$ respectively, where $\nu$ is the uncoded bit-rate, $R$ is the code-rate, $i$ is the number of iterations and $b$ is the number of bits used in the internal fixed point resolution of the decoder. Therefore, the NCG as a function of $R, O$ and $\nu$, denoted $\Psi(R, O, \nu)$, can be computed as $\Psi(R, O, \nu) \approx \max _{i, b} \Phi(R, i, b)$ subject to $\mathcal{O}(\nu, R, i, b)=O$. However, the latter optimization is not straightforward due to inherent complexity of $\mathcal{O}(\cdot)$ and $\Phi(\cdot)$. We suggest an approximation based on the observation that SD-FEC complexity is dominated by the complexity of the decoder which is approximately proportional to $i, b$ and the coded bit-rate $\nu / R$. This simplifies the complexity constraint to $O R i^{\prime} b^{\prime} \nu^{\prime}=O^{\prime} R^{\prime} i b \nu$, where $O^{\prime}$ is a reference complexity for a fixed set of parameters $\nu^{\prime}, i^{\prime}, b^{\prime}$ and $R^{\prime}$. In Fig. 10 we used $\nu=$ $\nu^{\prime}=100 \mathrm{~Gb} / \mathrm{s}, \bar{w}_{c}=4, N \approx 2 \cdot 10^{4}$ and $\Phi(R, i, b)$ estimated by computer simulation based on the LDPC code described in [79]. A similar tradeoff is expected for alternative architectures such as those described in [80].
} 
the symbol rate or the modulation order. Therefore the impact of a high $\mathrm{OH}$ FEC solution on the implementation complexity of other critical blocks such as ADCs, DACs, DSP blocks, etc., must be included in the analysis.

2) Structured Parity Check Matrix: A well structured parity check matrix can be used to mitigate the problem caused by the high interconnection complexity of parallel implementations of LDPC and TPC [80]. For instance, regular column-partition (RCP) [81], [82] combined with quasi-cyclic (QC) constraint was proposed in [42] for LDPC codes. Shift LDPC code also provides an efficient hardware oriented parity check matrix constraint [83]. We highlight that owing to the natural product code constraint, interconnection complexity in TPC is simpler than in most LDPC codes [84]-[86].

3) Variable Code Rates: The interconnection problem is exacerbated in rate-programmable FECs. A careful design is required to avoid (or minimize) further increase of area and power dissipation. The use of independent FECs allows architectures highly optimized for a fixed set of parameters to be designed. This approach avoids the power penalty by turning off the FEC blocks that are not used. However, its disadvantage is the high area that increases the cost of the chip. Therefore, an important effort is being directed towards designing programmable FEC architectures with low power and area penalties. A rate-adaptable architecture can be dynamically reconfigured to change the code-rate by varying the code-dimension or the code-length. To reduce complexity, code-rate variation is done by preserving the underlying structure of the parity check matrix, for example by adding or removing cyclic submatrices in a QC-LDPC code [79]. Thus, a well structured parity check matrix not only reduces the interconnection complexity but also provides a direct way to implement rate-adaptability. In this regard, LDPC codes have been shown to provide more flexibility than TPC codes [86].

4) Early Termination: The CG of SD-FEC increases as the number of iterations, $N_{i}$, increases. On the other hand, latency and power consumption grow with $N_{i}$. The number of iterations must be selected to provide a good tradeoff between complexity and performance (e.g., 5 to 15 iterations). For a given performance, the optimal (minimum) value of $N_{i}$ shall depend on the channel conditions. The value of $N_{i}$ required to achieve a certain performance reduces as OSNR increases. Furthermore, the number of iterations may vary at a certain OSNR due to different noise realizations. Then, $N_{i}$ should be dynamically adjusted to minimize power consumption and increase the FEC throughput. Several techniques to stop the decoding process have been reported in the past literature [87], [88]. Typically, these schemes are built upon two critera:

C1. Stop when the decoder has converged or a maximum number of iteration has been reached;

C2. Stop when the syndrome is zero or a maximum number of iterations has been reached.

Criterion $C 1$ avoids iterations once the soft-decision outputs are stable. This fact minimizes the probability of high values of $N_{i}$. When the errors in the received codeword can be corrected by the FEC, criterion $C 2$ minimizes the number of iterations. On the other hand, when the errors in the received codeword cannot be corrected, the maximum value of $N_{i}$ will be reached in $C 2$ based decoders. Therefore, a combination of $C 1$ and $C 2$ seems to be the best approach to minimize the number of iterations and power consumption.

5) Quantization: Fixed-point implementation of SD decoders plays an important role on both the performance and the complexity of FEC. The numbers of bits must be selected to minimize the effects of quantization and saturation. Typically, 4 and 5 bits uniform quantization (UQ) is adopted in practical VLSI implementations. Error floors caused by quantization effects with UQ [42] may be efficiently mitigated (or avoided) by using non-uniform quantization (NUQ) [89]. However, this improvement can be achieved at the expense of an increase of complexity of the arithmetic operations. Therefore, a careful analysis of UQ and NUQ must be carried out to assess the best tradeoff between complexity (e.g., arithmetic operations) and performance (e.g., number of bits).

\section{PRACTICAL IMPLEMENTATION TOPICS}

This section discusses low level ASIC implementation aspects with significant impact on the complexity and performance. These aspects are: (i) Floor planning, (ii) Placement, (iii) Clock tree synthesis (CTS), (iv) Routing, ( $v$ ) Timing signoff, (vi) Power dissipation, (vii) Power and SI, and (viii) Design for testability (DFT).

\section{A. Floor Planning}

Current generation coherent optical transceivers typically integrate in a single monolithic device functionality such as that exemplified in Figs. 1 and 2 and features such as those listed in Table II. Complexity may be in the 350 million gates range and beyond. Partitioning the design into blocks of manageable size is fundamental. There is tradeoff on the computation time required to process each block by the electronic design automation (EDA) tools and the integration time. As the number of blocks decreases so does the time and effort required for their integration. However, a reduced number of blocks requires managing large block sizes increasing the processing time for each individual block. The optimal size is in the range of 9 to 13.5 million gates. This is particularly important in DSP and FEC blocks, which perform a large amount of computation. This often leads to large data buses and complex interconnections. These must be managed beginning with the floor planning stage by adding physical restrictions (e.g., block shape, block area and port location). A square shape is commonly used as an starting point but sometimes it is not optimum for achieving the lowest power dissipation. The aspect ratio and the location of input/output (I/O) ports may have significant impact in the placement effort and the final power dissipation. Fig. 11 shows the top-level partitioning of a typical coherent optical transceiver as described in Figs. 1 and 2. Design must be partitioned in such a way as to minimize the number of physical ports per block. Some DSP algorithms such as BCD or FEC require some amount of local data storage. It is important to analyze the tradeoff between using random access memories (RAMs) and flip flops. Usually a large number of flip flops will increase the power dissipation 


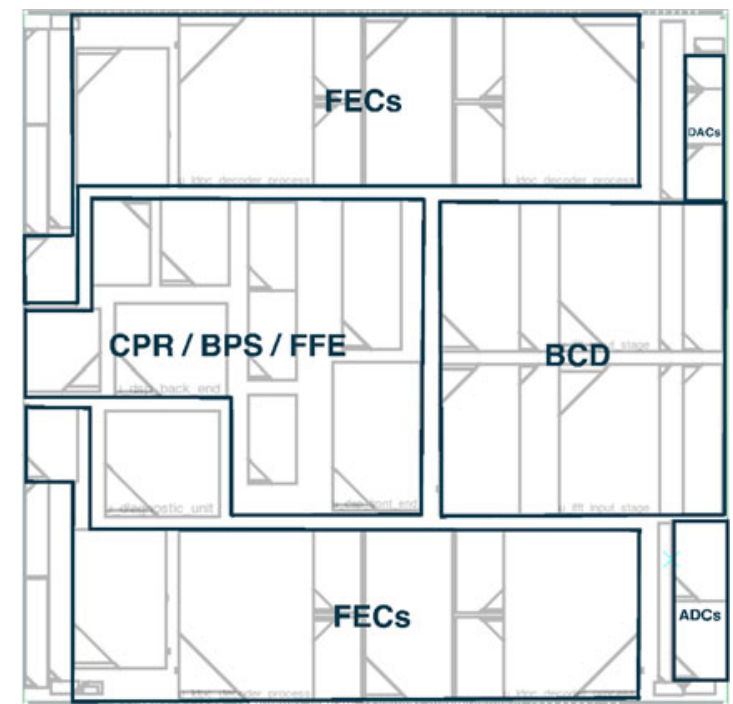

Fig. 11. Example of a top level partitioning for a typical optical coherent transceiver.

of the clock tree. It can also increase the complexity of the scan chains ${ }^{9}$ and require extra buffers for hold time fixes during static timing analysis (STA). On the other hand, RAMs generate physical restrictions to the place and route engines. This is exacerbated if memory compilers do not have the right combination of address and word length needed, restricting even more the design space. ${ }^{10}$ Advanced compilers support features targeting low power design such as power down, sleep mode, redundancy and transistors with high voltage threshold $\left(V_{t}\right)$ for peripheral logic, etc. If memories are in the data path (as required in some regular structures such as FFT and IFFT), they should be pre-placed at the floor planning stage in a fixed location following the data flow, thus reducing long interconnections and avoiding crisscrossing. This task may help reduce the parasitic capacitance of the wires and minimize the switching power component. Since not all ranges of frequencies are supported for the same RAM configuration, the memory may restrict the clock frequency of the logic computation surrounding it. This restriction can be avoided by using, for example, dual clock domains or even dual voltage domains.

\section{B. Placement}

At this stage the physical location of all components is defined. They remain essentially fixed during the rest of the physical design flow. The quality of the final results depends critically on achieving good placement. Typically, a significant increase in the number of cells compared to the post synthesis netlist occurs in this step. Buffer trees are built to distribute loads on nets with high fanout. It is possible to create hard or soft regions to guide placement. Moreover, it is possible to block certain areas. Placement can be driven to minimize congestion or to focus on

\footnotetext{
${ }^{9}$ Scan chains are sequential elements connected back to back to shift-in and shift-out test data. The goal of the scan chains is to make each node in the circuit controllable and observable.

${ }^{10}$ This is further exacerbated by the insertion of hardware to test defects on the memory blocks during the manufacturing process.
}

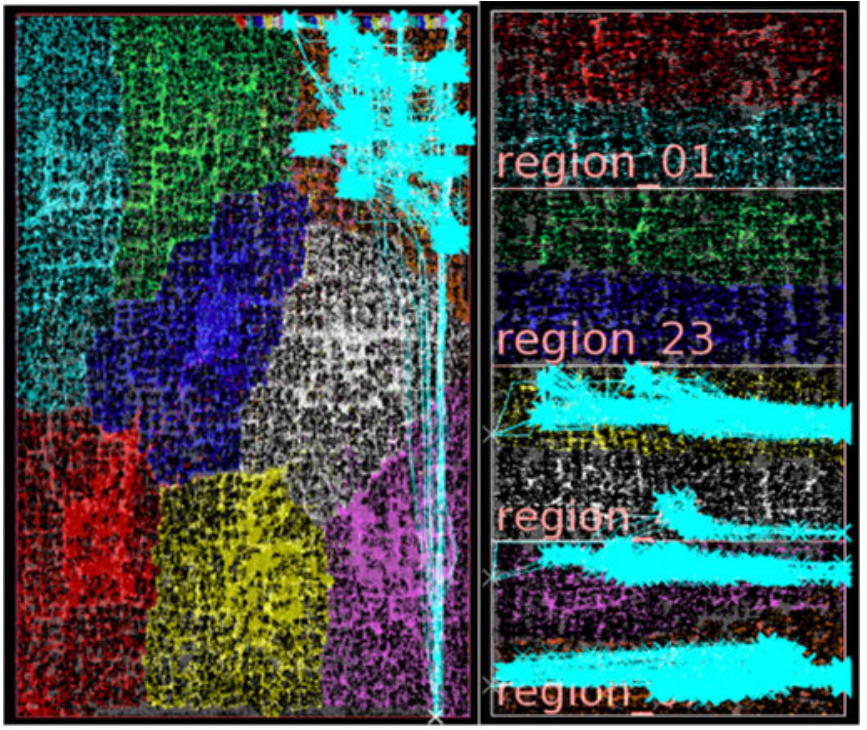

(a)

(b)

Fig. 12. Placement of a complex DSP block. (a) Flat placement (3.82 W). Fly lines show the input to output path through each group of taps from a filter. (b) Stratified placement $(2.55 \mathrm{~W}) \mathrm{I} / \mathrm{Os}$ ports placed at left and right plus additional place guides regions are used to guide data path.

timing. Manual placement (tiling) is used in regular data paths or in areas with large cell density. Initial placement attempts to achieve a homogeneous distribution and an area utilization ${ }^{11}$ of about $60 \%$. A certain percentage of spare cells is added during the placement. These cells can be used for small functional corrections at the very end stage in the design, before tapeout. These corrections are made by modifying interconnections based on metal layers only. If a new tape-out is needed to fix the problem, this technique may help reduce the manufacturing costs as only a few masks will change, without affecting the base layer masks. Fig. 12 shows two different floor plans of a DSP block. In Fig. 12(a) the tool automatically managed the location of the logic for each sub-block (flat placement) while in Fig. 12(b) the tool was restricted to follow a certain pattern in the location of logic and I/Os (stratified placement) based on the designer's knowledge of the functionality of the block. Different colors represent standard-cell density for each sub-block, while cyan regions show part of the wires connected to I/O ports with internal logic. In the flat placement, I/O ports are located at the top and bottom, while in the stratified placement the $\mathrm{I} / \mathrm{O}$ ports were located on the right side to minimize congestion and wire length. Different regions in the stratified placement indicate soft constraints used to guide the placement tools (there are four regions and each one comprises two sub-blocks). The total power dissipations for the flat and the stratified placements were 3.82 and $2.55 \mathrm{~W}$, respectively. This example shows that a human-assisted placement design is preferred for complex DSP blocks.

High power consumption of the BCD equalizer is mainly caused by the complex multiply-add operations required in

\footnotetext{
${ }^{11}$ Area utilization is the ratio between standard cells+macros area and the effective placement area.
} 
FFT/IFFT blocks. Custom multipliers in combination with a carefully selected subset of standard cells would enable tiling them manually in a very regular structure during placement, thus reducing the length of the interconnections. Placement must be guided in a different way to manage the complex interconnection of high-performance FEC parallel architectures. Clustering the processing unit elements and minimizing the drive strength of the buffers used in far interconnections between clusters are some of the options available to improve the placement of complex interconnection blocks such as iterative LDPC decoders.

\section{Clock Tree Synthesis}

Clock trees are one of the main sources of dynamic power usage. This makes CTS a critical power optimization step. Traditional automatic CTS design algorithms often work against the goal of power reduction by introducing more buffering than necessary. This is exacerbated by $(i)$ uncertainties in IC manufacturing and (ii) number of operating modes and power domains. A human assisted CTS design taking into account the implementation architecture of the involved blocks is needed to optimize power. Towards this end, CTS design must pursue strategies that lower the overall capacitance and minimize switching activity. Furthermore, CTS optimization should ideally trace back to the system level architecture design in order to improve the overall tradeoff between chip performance and complexity.

\section{Routing}

In blocks with intensive routing, such as SD-FEC, it is convenient to use the maximum stack of metals offered by the technology. Upper metal layers are used to built the power grid, medium layers for long interconnections, and low metal layers are preferred mostly for local interconnections. It is recommended to use via redundancy for reliability. Non default rules such as double width and double or triple space rules in clock nets may help minimize signal integrity (SI) effects such as cross coupling among clock nets and signal routing. Default rules represent a good starting point for signal nets. It is important to control the cell pin density in early stages to improve the pin accessibility and routability of the design. Over constrained design rules such as maximum transition or capacitance may result in high power dissipation.

\section{E. Timing Signoff}

The STA flow is set based on guidelines provided by the foundry and experience in taping out at the same technology node. The full chip is analyzed under process, voltage and temperature (PVT) variations for each operation mode. The quality of results is measured in terms of number cases and magnitude of: ( $i$ ) setup and hold timing violations, ( $i i$ ) signal and clock transitions, $($ iii $)$ minimum pulse width and period at clock pins of sequential elements and (iv) glitch or noise immunity. SI is verified in STA taking into account both crosstalk coupling noise and noise glitch effect. The former impacts delay negatively and the latter can upset adjacent logic circuitry. Noise effects are exacerbated at low voltage supplies, therefore effects such as dynamic voltage drop (DVD) must be considered in STA. In multimillion instance designs the voltage drop back annotation flow must be integrated in STA. This approach reduces risks associated with the above mentioned phenomena, which are difficult to predict accurately due to their dependence on system activity.

\section{F. Power Dissipation}

In CMOS technology the short circuit current and the current flow in interconnections determine dynamic power dissipation. Static power is dissipated even in stable states by the leakage currents of the transistors. Leakage exist unless the power supply is completely turned off. It can be reduced by using high $V_{t}$ cells as much as possible when large portions of the design are idle. Otherwise using large amounts of high $V_{t}$ cells may increase dynamic power due to the short circuit current in CMOS devices. It is also possible to automatically downsize the cells or swap $V_{t}$ types if timing is not degraded. In the same way, it is possible to remove hold buffers if there is enough hold margin. On the other hand, dynamic power depends on how large the amount of circuit operating at same time is. In data path intensive algorithms such as BCD, FFE, and CPR the entire circuits switch almost every clock, therefore, finding the lowest operating frequency is important. In designs where processing data cycle to cycle is not required, clock gating techniques are extensively used to reduce the switching activity on the clock path. Alternatively, the sharing operator technique is commonly used when there are several clock cycles available to process the incoming data. This allows arithmetic processing elements such as multipliers and adders to be reused. From the technology point of view, choosing advanced technologies such as FinFet transistors is a good option to reduce power in comparison to planar transistors. From the point of view of the algorithms some tradeoffs need to be analyzed. For instance, reducing hardware by increasing frequency not necessarily results in power savings.

To meet power specifications it is necessary to have an early estimation of power consumption. This allows designers to react during the algorithm and architecture design phase. With modern EDA tools power can be estimated at register transfer level (RTL). These tools use the information from the technology libraries plus generic parasitic models extracted from a real layout in combination with the toggling information provided by the RTL simulation. The fast estimation of this approach allows different architectures to be studied and refinements to be made. Iteration time is low as the layout flow is skipped in this loop. The accuracy of these power numbers is good enough to make decisions and select among different architectures.

Final power estimations are performed post layout, having at this point the physical information of the routing. The accuracy of the results is set by the quality of the inputs used into the power calculation signoff tool. The inputs are: $(i)$ standard parasitic exchange format (SPEF) files, (ii) timing libraries, and (iii) value change dump (VCD) files. SPEF files provide parasitic information with all the capacitances extracted using signoff parasitic extraction tools. Timing libraries include power 
information and they are selected based on the signoff criteria determined by the application. VCD files provide the toggle information needed to analyze the dynamic power components. The VCD is typically dumped from gate level simulations under real conditions. These simulations annotate the cell and wire delays extracted from STA signoff tools. Using the real delay of the cells and wires in the simulation is important to capture the glitches that occur in the data path. These glitches have important impact on the toggle count. Omitting glitch effects in DSP systems results in optimistic power estimations.

\section{G. Power and SI}

Power integrity is related to the stability of power sources. Ideally, the maximum current can be delivered at any rate to the power distribution network (PDN) while the voltage at the output of the power source remains constant. Unfortunately, real power sources suffer from degradations due to resistance, reactance and resonance, called the 3Rs. The voltage degradation in the PDN due to the resistance increases with wire lengths. The later generate a static voltage drop caused by the average current flowing through the PDN which is known as IR drop. The voltage drop in the PDN due to the reactance is a DVD proportional to the inductance of the PDN and the temporal variations of the current flowing through the PDN. Therefore, a high inductance and a fast rate of change of the current exacerbates this degradation. On the other hand, the resonance is intrinsic to the system. The PDN can be represented by an $R L C$ circuit with a frequency of resonance $f_{r}$. At this frequency the impedance seen from the switching circuits towards to the voltage source is maximum [90], [91]. This situation must be avoided by ensuring that this frequency is far enough from the frequency of operation of the circuit. Inserting additional capacitance is useful, usually on-chip decoupling-caps and integrated capacitors for package are used for such purpose. For instance, circuits such as FFT/IFFT blocks which require large amount of current in a small fraction of time and operate at frequencies close to the resonant frequency of the PDN may generate power integrity issues, such as ground bouncing. A proper control of the frequency of resonance or the frequency of operation helps to avoid power integrity issues. Toward this end, a complete model of the entire PDN is required to do an accurate analysis (e.g., model must include board, package and die impedances)

Power integrity noise in power and ground lines is caused by simultaneous switching of large numbers of gates and registers. Therefore, minimizing the impedance of the PDN (on board, on package and on chip) helps reduce the problem. Impedance minimization can be achieved by reducing the PDN resistance and inductance or by using decoupling capacitors. However, the later has equivalent series resistance and equivalent series inductance which also cause resonant behavior.

Finally, SI requires preserving the bandwidth, reducing latency, minimizing noise, and reducing power dissipation of interconnects. Coupling noise has three primary deleterious effects: (i) Functional failure, (ii) Glitch power consumption, and (iii) Delay uncertainty. Circuit level methodologies for
SI include the design of tapered buffers, repeater insertion, shielding, gate and wire sizing, wire spacing, signal rerouting, and wire reordering (e.g., see [92] for a thorough analysis of these topics).

\section{H. Design for Testability}

Detecting failures in integrated circuits at submicron technology nodes has become a major challenge. Large and complex designs require both structural DFT and $a d-h o c$ techniques to detect manufacturing defects and to test performance of the design. A combination of dedicated hardware and embedded firmware is needed to ensure the highest level of test quality in complex system on chips (SoCs) for optical coherent transceivers. Design size, operation modes, flip-flop count, test time and power dissipation are parameters commonly explored when designing a DFT strategy. Core test wrapping methodology has been extensively used to give more flexibility and control over the observability points [93]. These core wrappers can be selected independently to receive testing patterns and this way put a specific region of the chip under test.

$A d$-hoc DFT techniques are used for debugging and functional verification. The transceiver typically incorporates a serial peripheral interface which provides access to registers used to control the device operation, read or write parameters and coefficients, and read status signals. It also has a diagnostic unit (DU), which provides a host of observability and controllability features used for testing, characterization, and channel diagnostics. For example, the DU captures data from chip blocks such as the ADC, FFE, CPR, etc., in real time and then it is accessed externally at lower speed. In this way, internal data from almost any point in the chip can be captured and analyzed by software.

\section{CONCLUSION}

Coherent transceivers are among the most complex devices designed by the semiconductor industry. They implement sophisticated signal processing, coding, framing and mapping functions at ultra high speed, meeting high performance and low power dissipation requirements. Flexible transceivers able to operate in software defined networks at rates up to $200 \mathrm{~Gb} / \mathrm{s}$ per wavelength are now commercially available. The semiconductor industry is currently actively working on transceivers operating at $400 \mathrm{~Gb} / \mathrm{s}$ and beyond. The DSP revolution in optical communications is expected to continue in the future with the application of even more advanced techniques. This will result in increases in data rate, spectral efficiency and flexibility of the network as well as in significant cost reductions.

\section{REFERENCES}

[1] X. Liu, S. Chandrasekhar, and P. Winzer, "Digital signal processing techniques enabling multi-Tb/s superchannel transmission," IEEE Signal Process. Mag., vol. 31, no. 2, pp. 16-24, Mar. 2014.

[2] M. Kuschnerov et al., "Advances in signal processing," presented at the European Conf. Exhibition Optical Communication, Amsterdam, The Netherlands, 2012, Paper We.2.A.1.

[3] X. Zhou and L. Nelson, "Advanced DSP for $400 \mathrm{~Gb} / \mathrm{s}$ and beyond optical networks," J. Lightw. Technol., vol. 32, no. 16, pp. 2716-2725, Aug. 2014

[4] M. Kuschnerov et al., "DSP for coherent single-carrier receivers," $J$ Lightw. Technol., vol. 27, no. 16, pp. 3614-3622, Aug. 2009. 
[5] M. Jinno et al., "Distance-adaptive spectrum resource allocation in spectrum-sliced elastic optical path network," IEEE Signal Process. Mag., vol. 48, no. 8, pp. 138-145, Aug. 2010.

[6] P. Layec et al., "Elastic optical networks: The global evolution to software configurable optical networks," J. Bell Labs Tech., vol. 18, no. 3, pp. 133-155, 2013.

[7] N. Sambo et al., "Next generation sliceable bandwidth variable transponders," IEEE Signal Process. Mag., vol. 53, no. 2, pp. 163-171, Feb. 2015.

[8] A. Barbieri, G. Colavolpe, and G. Caire, "Joint iterative detection and decoding in the presence of phase noise and frequency offset," IEEE Trans. Commun., vol. 55, no. 1, pp. 171-179, Jan. 2007.

[9] M. A. Castrillon et al. (2015). On the performance of joint iterative detection and decoding in coherent optical channels with laser frequency fluctuations," Opt. Fiber Technol. [Online]. Available: http://www.sciencedirect.com/science/article/pii/S1068520015000474

[10] O. Gerstel et al., "Elastic optical networking: A new dawn for the optical layer?" IEEE Signal Process. Mag., vol. 50, no. 2, pp. s12-s20, Feb. 2012.

[11] M. Jinno et al., "Spectrum-efficient and scalable elastic optical path network: Architecture, benefits, and enabling technologies," IEEE Signal Process. Mag., vol. 47, no. 11, pp. 66-72, Nov. 2009.

[12] G. Gho and J. Kahn, "Rate-adaptive modulation and coding for optical fiber transmission systems," J. Lightw. Technol., vol. 30, no. 12, pp. 1818-1828, Jun. 2012

[13] G. Tzimpragos et al., "A survey on FEC codes for $100 \mathrm{G}$ and beyond optical networks," IEEE Commun. Surveys Tuts., to be published.

[14] E. Ip and J. Kahn, "Fiber impairment compensation using coherent detection and digital signal processing," J. Lightw. Technol., vol. 28, no. 4, pp. 502-519, Feb. 2010.

[15] D. Crivelli et al., "Architecture of a single-chip $50 \mathrm{~Gb} / \mathrm{s}$ DP-QPSK/BPSK transceiver with electronic dispersion compensation for coherent optical channels," IEEE Trans. Circuits Syst. I, Reg. Papers, vol. 61, no. 4, pp. 1012-1025, Apr. 2014.

[16] C. Laperle, "Advances in high speed ADC, DAC, and DSP for optical transceivers," in Proc. Opt. Fiber Commun. Conf., Anaheim, CA, USA, 2013, Paper OTh1F.5.

[17] N. Stojanovic, Y. Zhao, and C. Xie, "Feed-forward and feedback timing recovery for Nyquist and faster than Nyquist systems," presented at the Optical Fiber Communication Conf., San Francisco, CA, USA, 2014 Paper Th3E.3.

[18] D. Crivelli, H. Carrer, and M. Hueda, "Adaptive digital equalization in the presence of chromatic dispersion, PMD, and phase noise in coherent fiber optic systems," in Proc. IEEE GLOBECOM, 2004, vol. 4, pp. 2545-2551.

[19] T. Pfau, S. Hoffmann, and R. Noe, "Hardware-Efficient coherent digital receiver concept with feedforward carrier recovery for M-QAM constellations," J. Lightw. Technol., vol. 27, no. 8, pp. 989-999, Apr. 2009.

[20] P. Gianni et al., "Compensation of laser frequency fluctuations and phase noise in 16-QAM coherent receivers," IEEE Photon. Technol. Lett., vol. 25, no. 5, pp. 442-445, Mar. 2013.

[21] K. Onohara et al., "Soft-decision-based forward error correction for 100 Gb/s transport systems," IEEE J. Sel. Topics Quantum Electron., vol. 16, no. 5, pp. 1258-1267, Sep. 2010.

[22] M. Taylor, "Phase estimation methods for optical coherent detection using digital signal processing," J. Lightw. Technol., vol. 27, no. 7, pp. 901-914, Apr. 2009.

[23] P. Gianni et al., "A new parallel carrier recovery architecture for intradyne coherent optical receivers in the presence of laser frequency fluctuations," in Proc. IEEE GLOBECOM, 2011, pp. 1-6.

[24] G. P. Agrawal, Nonlinear Fiber Optics (ser. Optics and Photonics). San Diego, CA, USA: Academic, 2006.

[25] M. Magarini et al., "Empirical modeling and simulation of phase noise in long-haul coherent optical transmission systems," Opt. Exp., vol. 19, no. 23 , pp. 22 455-22 461, Nov. 2011.

[26] Z. Tao et al., "Simple fiber model for determination of XPM effects," $J$. Lightw. Technol., vol. 29, no. 7, pp. 974-986, Apr. 2011.

[27] S. Kumar, "Analysis of nonlinear phase noise in coherent fiber-optic systems based on phase shift keying," J. Lightw. Technol., vol. 27, no. 21, pp. 4722-4733, Nov. 2009.

[28] T. Pfau, S. Hoffmann, and R. Noe, "Coherent optical 25.8-Gb/s OFDM transmission over 4160-km SSMF," J. Lightw. Technol., vol. 26, no. 1, pp. 6-15, Feb. 2008

[29] A. Diaz et al., "Analysis of back-propagation and RF pilot-tone based nonlinearity compensation for a $9 \times 224 \mathrm{~Gb} / \mathrm{s}$ POLMUX-16QAM system," presented at the Optical Fiber Communication Conf., Los Angeles, CA, USA, 2012, Paper OTh3C.5.
[30] R. Soriano et al., "Chromatic dispersion estimation in digital coherent receivers,” J. Lightw. Technol., vol. 29, no. 11, pp. 1627-1637, May 2011.

[31] S. Qi, A. Lau, and L. Chao, "Fast and robust blind chromatic dispersion estimation using auto-correlation of signal power waveform for digital coherent systems," J. Lightw. Technol., vol. 31, no. 2, pp. 306-312, Jan. 2013.

[32] J. E. Mazo, "Faster-than-Nyquist signaling," J. Bell Labs Tech., vol. 54, no. 8 , pp. 1451-1462, 1975.

[33] X. Li et al., "Electronic post-compensation of WDM transmission impairments using coherent detection and digital signal processing," Opt. Exp., vol. 16, no. 2, pp. 880-888, 2008.

[34] Z. Maalej et al., "Reduced complexity back-propagation for optical communication systems," presented at the Optical Fiber Communication Conf., Los Angeles, CA, USA, 2012, Paper JW2A.63.

[35] D. Millar et al., "Mitigation of fiber nonlinearity using a digital coherent receiver," IEEE J. Sel. Topics Quantum Electron., vol. 16, no. 5, pp. 1217-1226, Sep. 2010.

[36] C.-Y. Lin et al., "Adaptive digital back-propagation for optical communication systems," presented at the Optical Fiber Communication Conf., San Francisco, CA, USA, 2014, Paper M3C.4.

[37] Z. Tao et al., "Multiplier-free intrachannel nonlinearity compensating algorithm operating at symbol rate," J. Lightw. Technol., vol. 29, no. 17, pp. 2570-2576, Sep. 2011.

[38] Q. Zhuge et al., "Aggressive quantization on perturbation coefficients for nonlinear pre-distortion," presented at the Optical Fiber Communication Conf., San Francisco, CA, USA, 2014, Paper Th4D.7.

[39] B. Smith et al., "Staircase codes: FEC for $100 \mathrm{~Gb} / \mathrm{s}$ OTN," J. Lightw. Technol., vol. 30, no. 1, pp. 110-117, Jan. 2012.

[40] D. Chang et al., "LDPC convolutional codes using layered decoding algorithm for high speed coherent optical transmission," presented at the Optical Fiber Communication Conf., Los Angeles, CA, USA, 2012, Paper OW1H.4.

[41] L. Nelson et al., "WDM performance and multiple-path interference tolerance of a real-time $120 \mathrm{Gbps}$ Pol-Mux QPSK transceiver with soft decision FEC," presented at the Optical Fiber Communication Conf., Los Angeles, CA, USA, 2012, Paper NTh1I.5.

[42] D. Morero et al., "Non-concatenated FEC codes for ultra-high speed optical transport networks," in Proc. IEEE GLOBECOM, 2011, pp. 1-5.

[43] Y. Miyata et al., "UEP-BCH product code based hard-decision FEC for $100 \mathrm{~Gb} / \mathrm{s}$ optical transport networks," presented at the Optical Fiber Communication Conf., Los Angeles, CA, USA, 2012, Paper JW2A.7.

[44] I. Djordjevic, L. Xu, and T. Wang, "Reverse concatenated coded modulation for high-speed optical communication," IEEE Photon. J., vol. 2, no. 6, pp. 1034-1039, Dec. 2010.

[45] K. Liu et al., "Quasi-cyclic LDPC codes: Construction and rank analysis of their parity-check matrices," in Proc. Inform. Theory Appl. Workshop, San Diego, CA, USA, 2012, pp. 227-233.

[46] S. Song et al., "A unified approach to the construction of binary and nonbinary quasi-cyclic LDPC codes based on finite fields," IEEE Trans. Commun., vol. 57, no. 1, pp. 84-93, Jan. 2009.

[47] L. Zhang et al., "Quasi-cyclic LDPC codes on cyclic subgroups of finite fields," IEEE Trans. Commun., vol. 59, no. 9, pp. 2330-2336, Sep. 2011.

[48] K. Sugihara et al., "A spatially-coupled type LDPC code with an NCG of $12 \mathrm{~dB}$ for optical transmission beyond $100 \mathrm{~Gb} / \mathrm{s}$," presented at the Optical Fiber Communication Conf., Anaheim, CA, USA, 2013, Paper OM2B.4.

[49] J. Li et al., "Algebraic quasi-cyclic LDPC codes: Construction, low errorfloor, large girth and a reduced-complexity decoding scheme," IEEE Trans. Commun., vol. 62, no. 8, pp. 2626-2637, Aug. 2014

[50] F. Buchali et al., "Implementation of 64QAM at 42.66 GBaud using 1.5 samples per symbol DAC and demonstration of up to $300 \mathrm{~km}$ fiber transmission," presented at the Optical Fiber Communication Conf., San Francisco, CA, USA, 2014, Paper M2A.1.

[51] Digital Video Broadcasting, ETSI EN Standard 302 307, Rev. 1.2.1, 2009

[52] M. Castrillon, D. Morero, and M. Hueda, "A new cycle slip compensation technique for ultra high speed coherent optical communications," in Proc. IEEE Photon. Conf., Burlingame, CA, USA, Sep. 2012, pp. 175-176, Paper MU2.

[53] H. Zhang et al. "Cycle slip mitigation in POLMUX-QPSK modulation," presented at the Optical Fiber Communication Conf., Los Angeles, CA, USA, 2011, Paper OMJ7.

[54] T. Richardson, "Error floors of LDPC codes," presented at the 41th Annual Allerton Communication Control Computing Conf., Monticello, IL, USA, 2003.

[55] D. Morero and M. Hueda, "Novel serial code concatenation strategies for error floor mitigation of low-density parity-check and turbo product 
codes," Can. J. Elect. Comput. Eng., vol. 36, no. 2, pp. 52-59, Spring 2013.

[56] D. Schneider, "The microsecond market," IEEE Spectrum, vol. 49, no. 6, pp. 66-81, Jun. 2012.

[57] J. Geyer et al., "Practical implementation of higher order modulation beyond 16-QAM," presented at the Optical Fiber Communication Conf., Los Angeles, CA, USA, 2015, Paper Th1B.1.

[58] C. Campopiano and B. Glazer, "A coherent digital amplitude and phase modulation scheme," IRE Trans. Commun. Syst., vol. 10, no. 1, pp. 90-95, Mar. 1962.

[59] F. Schreckenbach et al., "Optimization of symbol mappings for bitinterleaved coded modulation with iterative decoding," IEEE Commun. Lett., vol. 7, no. 12, pp. 593-595, Dec. 2003.

[60] S. ten Brink, "Convergence of iterative decoding," Electron. Lett., vol. 35, no. 10, pp. 806-808, May 1999.

[61] G. Forney et al., "Efficient modulation for band-limited channels," IEEE J. Select. Areas Commun., vol. 2, no. 5, pp. 632-647, Sep. 1984.

[62] M. Karlsson and E. Agrell, "Which is the most power-efficient modulation format in optical links?" Opt. Exp., vol. 17, no. 13, pp. 10 814-10 819, Jun. 2009

[63] M. Karlsson and E. Agrell, "Four-dimensional optimized constellations for coherent optical transmission systems," presented at the European Optical Communication Conf., Torino, Italy, 2010, Paper We.8.C.3.

[64] L. Coelho and N. Hanik, "Global optimization of fiber-optic communication systems using four-dimensional modulation formats," presented at the European Optical Communication Conf., Geneva, Switzerland, 2011, Paper Mo.2.B.4.

[65] T. A. Eriksson et al., "Experimental investigation of a four-dimensional 256-ary lattice-based modulation format," presented at the Optical Fiber Communication Conf., Los Angeles, CA, USA, 2015, Paper W4K.3.

[66] M. Magarini et al., "Pilot-symbols-aided carrier-phase recovery for 100G PM-QPSK digital coherent receivers," IEEE Photon. Technol. Lett., vol. 24, no. 9, pp. 739-741, May 2012.

[67] F. Yu et al., "Soft-decision LDPC turbo decoding for DQPSK modulation in coherent optical receivers," presented at the European Optical Communication Conf., Geneva, Switzerland, 2011, Paper We.10.P1.70.

[68] M. A. Castrillon, D. A. Morero, and M. R. Hueda. (2012). Joint demapping and decoding for DQPSK optical coherent receivers. CoRR. vol. abs/1206.4914, [Online]. Available: http://arxiv.org/abs/1206.4914

[69] C. B. Czegledi, E. Agrell, and M. Karlsson, "Symbol-by-symbol joint polarization and phase tracking in coherent receivers," presented at the Optical Fiber Communication, Los Angeles, CA, USA, 2015, Paper W1E.3.

[70] N. Irukulapati et al., "Stochastic digital backpropagation," IEEE Trans. Commun., vol. 62, no. 11, pp. 3956-3968, Nov. 2014.

[71] S. O. Arik, K.-P. Ho, and J. M. Kahn, "Optical network scaling: Roles of spectral and spatial aggregation," Opt. Exp., vol. 22, no. 24, pp. 29 868-29 887, Dec 2014.

[72] D. Hillerkuss, Single-Laser Multi-Terabit/s Systems (ser. XVIII), 184 S. Karlsruhe, Germany: KIT Scientific Publishing, 2013.

[73] P. Winzer, "High-spectral-efficiency optical modulation formats," $J$. Lightw. Technol., vol. 30, no. 24, pp. 3824-3835, Dec. 2012.

[74] J. Anderson, F. Rusek, and V. Owall, "Faster-than-Nyquist signaling," Proc. IEEE, vol. 101, no. 8, pp. 1817-1830, Aug. 2013.

[75] G. Raybon et al., "Single-carrier and dual-carrier 400-Gb/s and 1.0-Tb/s transmission systems," presented at the Optical Fiber Communication Conf., San Francisco, CA, USA, 2014, Paper Th4F.1.

[76] G. Raybon, "High symbol rate transmission systems for data rates from $400 \mathrm{~Gb} / \mathrm{s}$ to $1 \mathrm{~Tb} / \mathrm{s}$," presented at the Optical Fiber Communication Conf., Los Angeles, CA, USA, 2015, Paper M3G.1.

[77] P. Duhamel, "Algorithms meeting the lower bounds on the multiplicative complexity of length- $2^{n}$ DFTs and their connection with practical algorithms," IEEE Trans. Acoust. Speech Signal Process., vol. 38, no. 9, pp. 1504-1511, Sep. 1990.

[78] O. E. Agazzi, "Design trade-offs in practical ASIC implementations," presented at the Optical Fiber Communication Conf., Los Angeles, CA, USA, 2015, Paper Th1B.3.

[79] D. Morero et al., "Non-concatenated FEC codes for ultra-high speed optical transport networks," U.S. Patent 8918 694, Dec. 23, 2014

[80] Z. Wang, Z. Cui, and J. Sha, "VLSI design for low-density parity-check code decoding," IEEE Circuits Syst. Mag., vol. 11, no. 1, pp. 52-69, Jan./Mar. 2011.

[81] D. Morero, G. Corral-Briones, and M. Hueda, "Parallel architecture for decoding LDPC codes on high speed communication systems," in Proc. Argentine School Micro-Nanoelectronics Technol. Appl., Buenos Aires, Argentina, 2008, pp. 107-110.
[82] L. Liu and C.-J. Shi, "Sliced message passing: High throughput overlapped decoding of high-rate low-density parity-check codes," IEEE Trans. Circuits Syst. I, Reg. Papers, vol. 55, no. 11, pp. 3697-3710, Dec. 2008.

[83] J. Sha et al., "Multi-Gb/s LDPC code design and implementation," IEEE Trans. Very Large Scale Integr. Syst., vol. 17, no. 2, pp. 262-268, Feb. 2009.

[84] C. Jego, P. Adde, and C. Leroux, "Full-parallel architecture for turbo decoding of product codes," Electron. Lett., vol. 42, no. 18 , pp. 1052-1053, Aug. 2006.

[85] R. Pyndiah et al., "Near optimum decoding of product codes," in Proc. IEEE GLOBECOM, 1994, vol. 1, pp. 339-343.

[86] S. Dave et al., "Soft-decision forward error correction in a 40-nm ASIC for 100-Gbps OTN applications," in Proc. Opt. Fiber Commun. Conf., Los Angeles, CA, USA, 2011, Paper JWA014.

[87] Y.-H. Chen et al., "A channel-adaptive early termination strategy for LDPC decoders," in Proc. IEEE Workshop Signal Process. Syst., Tampere, Finland, 2009, pp. 226-231.

[88] W. Stoye, "LDPC iteration control by partial parity check," in Proc. IEEE Int. Conf. Ultra-Wideband, Vancouver, BC, Canada, 2009, pp. 602-605.

[89] X. Zhang and P. Siegel, "Quantized iterative message passing decoders with low error floor for LDPC codes," IEEE Trans. Commun., vol. 62, no. 1, pp. 1-14, Jan. 2014

[90] R. Nair and D. Bennett, Power Integrity Analysis and Management for Integrated Circuits (Prentice Hall Signal Integrity Library), Englewood Cliffs, NJ, USA: Prentice-Hall, 2010.

[91] M. Popovich, A. Mezhiba, and E. Friedman, Power Distribution Networks With On-Chip Decoupling Capacitors. New York, NY, USA: Springer, 2007.

[92] E. Salman and E. G. Friedman, High Performance Integrated Circuit Design. New York, NY, USA: McGraw Hill, 2012.

[93] P. Girard, N. Nicolici, and X. Wen, Power-Aware Testing and Test Strategies for Low Power Devices (SpringerLink: Bücher), New York, NY, USA: Springer, 2010.

Damián A. Morero was born in Córdoba, Argentina. He received the degree in electronic engineering with honors and the Ph.D. degree in engineering science from the National University of Cordoba (UNC), Cordoba, Argentina. In 2003 and 2005, he received the Academic Excellence Award from the Engineers Association of Cordoba Argentina and the UNC, respectively. From 2006 to 2009, he received Ph.D. Fellowships from the Secretary of Science and Technology (SeCyT), Argentina. He is currently the Project Director at ClariPhy Argentina S.A. where he has been engaged in the research and development of Error Correction Coding schemes and Digital Signal Processing algorithms for high speed optical communications and data storage. His research interests include coding, information theory, signal processing, computer science and VLSI design.

Mario A. Castrillón received the Electronic Engineer degree from the National Technological University, Córdoba, Argentina, in 2009. In the same year, he joined the Digital Communications Research Laboratory, Department of Electronic Engineering, National University of Córdoba, Córdoba, Argentina, where he is currently working toward the Ph.D. degree in engineering sciences. His research interests include coding, information theory, and signal processing. From 2009 to 2013, he received the Ph.D. Fellowship from the Secretary of Science and Technology, Argentina. He received a Type-II Grant awarded by National Scientific and Technical Research Council, Argentina.

Alejandro Aguirre was born in Corrientes, Argentina. He received the Electronic Engineer degree from the National University of Córdoba, Argentina, in 2011. In 2009, he joined ClariPhy Argentina S.A. where he started working on high speed analog circuit design and later moved to physical design of mixed signal integrated circuits. Since 2010, he has been responsible for the physical design of several generations of optical coherent transceivers at speeds of 10 , 40, 100 , and $200 \mathrm{~Gb} / \mathrm{s}$. 
Mario R. Hueda was born in Jujuy, Argentina. He received the Electrical and Electronic Engineer degree in 1994, and the Ph.D. degree in 2002, both from the National University of Cordoba, Argentina. Since 1997, he has been with the Digital Communications Research Laboratory at the Department of Electronic Engineering, National University of Cordoba, Argentina. He is also with CONICET (National Scientific and Technological Research Council of Argentina). His research interests include digital communications, signal processing, and synchronization. He has 15 patents issued or pending, and has published more than 60 technical papers in journals and conferences.
Oscar E. Agazzi received the Ph.D. degree in electronic engineering from the University of California at Berkeley, Berkeley, CA, USA. He is the Senior Vice-President and Chief Systems Architect at ClariPhy Communications, Inc. $\mathrm{He}$ is the Chief Architect of the ClariPhy family of coherent as well as direct detection optical transceivers. Prior to joining ClariPhy, he worked at Broadcom Corporation and at Lucent Technologies Bell Laboratories. He has more than 150 patents issued or pending, and has published more than 60 technical papers in journals and conferences. He is a Lucent Technologies Bell Labs Fellow. 